\title{
Polarization and Public Health: Partisan Differences in Social Distancing during the Coronavirus Pandemic
}

\author{
Hunt Allcott, New York University, Microsoft Research, and NBER* \\ Levi Boxell, Stanford University \\ Jacob Conway, Stanford University \\ Matthew Gentzkow, Stanford University and NBER \\ Michael Thaler, Harvard University \\ David Yang, Harvard University and NBER
}

July 2020

\begin{abstract}
We study partisan differences in Americans' response to the COVID-19 pandemic. Political leaders and media outlets on the right and left have sent divergent messages about the severity of the crisis, which could impact the extent to which Republicans and Democrats engage in social distancing and other efforts to reduce disease transmission. We develop a simple model of a pandemic response with heterogeneous agents that clarifies the causes and consequences of heterogeneous responses. We use location data from a large sample of smartphones to show that areas with more Republicans engaged in less social distancing, controlling for other factors including public policies, population density, and local COVID cases and deaths. We then present new survey evidence of significant gaps at the individual level between Republicans and Democrats in self-reported social distancing, beliefs about personal COVID risk, and beliefs about the future severity of the pandemic.
\end{abstract}

\footnotetext{
*E-mail: hunt.allcott@nyu.edu, lboxell@stanford.edu, jcconway@stanford.edu, gentzkow@stanford.edu, michaelthaler@g.harvard.edu, davidyang@fas.harvard.edu. We thank Victoria Pu for research assistance. We thank SafeGraph for providing access to the data and the SafeGraph COVID-19 response community for helpful input. We thank Lubos Pastor along with seminar participants at Stanford University, Harvard University, and the University of Chicago for their comments and suggestions. We acknowledge funding from the Stanford Institute for Economic Policy Research (SIEPR), the John S. and James L. Knight Foundation, the Sloan Foundation, the Institute for Humane Studies, and the National Science Foundation (grant number: DGE-1656518). For our survey, we registered a pre-analysis plan on the AEA Registry, with ID AEARCTR-0005632. This study was approved by IRBs at NYU (IRB-FY2020-4331), Harvard (IRB17-1725), and Stanford (eProtocol 42883).
} 


\section{Introduction}

Public messaging in the US during the coronavirus pandemic has diverged sharply along partisan lines. President Trump and other Republican officials have sometimes downplayed the severity of the crisis, while Democratic leaders have given more emphasis to its dangers (Beauchamp 2020; Stanley-Becker and Janes 2020; Coppins 2020; McCarthy 2020). Similar divisions appear in partisan media (Aleem 2020; Kantrowitz 2020).

Nationwide surveys mirror the partisan divisions in elite messaging —with Democrats reporting more concern about COVID-19 and higher levels of social distancing than Republicans (see Figure 1). However, Democratic areas have also had more coronavirus cases and implemented stay-athome policies earlier. The raw differences observed on surveys could simply be the expected result of local differences in risk or regulation. Furthermore, prior evidence shows that apparent partisan gaps in beliefs can shrink substantially when there are moderate incentives for accuracy (Bullock et al. 2015; Prior et al. 2015). Beliefs about the number of casualties in Iraq or the presidential approval rating have, for most people, few direct consequences. Beliefs about the severity of the pandemic and choosing whether to social distance, on the other hand, may be a matter of life or death. We ask whether partisan gaps persist in the face of these large incentives.

In this paper, we combine GPS location data from a large sample of smartphones with a new survey to study partisan differences in the response to COVID-19. The GPS data are collected by the company SafeGraph, and record daily and weekly visits to points of interest (POIs), including restaurants, hotels, hospitals, and many other public and private businesses. Our primary analysis focuses on the period from January 27, 2020 to July 12, 2020.

We present a simple model that clarifies the potential causes and consequences of divergent social-distancing behavior. It combines a standard epidemiological model of a pandemic with an economic model of optimizing behavior by heterogeneous agents. The model clarifies that divergent responses between groups need not be inefficient. One group might engage in less social distancing because their costs of distancing are greater (e.g., they would lose more income) or because their benefits of distancing are smaller (e.g., they are at lower risk of infection). However, differences in behavior resulting from divergent beliefs of otherwise similar agents do suggest systematic inefficiency, as optimizing based on different beliefs means that the marginal costs of social distancing are not equated across people. Achieving a given level of social distancing in society will be more costly when otherwise similar agents have heterogeneous beliefs. 
Our main GPS results show that the strong partisan differences in social distancing behavior that emerged with the rise of COVID-19 are not merely an artifact of differences in public policies or observed risks. Controlling for state-time fixed effects to account for heterogenous policy responses by state governments only attenuates the partisan gap slightly. Including controls to proxy for local policy, health, weather, and economic variables interacted flexibly with time attenuates the gap more substantially, but it remains statistically and economically significant. After including our full set of controls, we estimate that moving from the 10th to the 90th percentile of Republican county vote share is associated with 11.5 and 15.2 percent increases in the number of POI visits during the weeks of April 6 and May 11, when social distancing and partisan gaps are at their respective peaks.

Our findings are robust to the inclusion or exclusion of control variables, excluding states with early COVID-19 outbreaks, or dropping highly populated counties. Replacing the continuous measure of partisanship with discrete indicators for portions of the Republican vote share distribution or restricting the sample to counties from certain portions of the distribution does not change our qualitative conclusions. Furthermore, there is no evidence of a similar partisan gap during the same period in 2019 conditional on the same set of controls. We find similar evidence of a partisan gap at the voting precinct level, but focus on county-level analyses due to limitations facing the precinct specification (see footnote 14).

To complement the data showing county-level differences in behavior, we use a nationallyrepresentative survey to show that individual behavior and beliefs about social distancing are partisan. We collect participants' demographics (including party affiliation), beliefs regarding the efficacy of social distancing, self-reported distancing due to COVID-19, and predictions about future COVID-19 cases. Compared to Republicans, we find that Democrats believe the pandemic is more severe and report a greater reduction in contact with others. In our survey, we also randomly vary whether predictions about future COVID-19 cases are incentivized. We do not find evidence that incentives reduce the partisan gap, suggesting that these predictions are less likely to be due to partisan cheerleading (as in Bullock et al. 2015 and Prior et al. 2015) and more likely to reflect true differences in beliefs. These partisan gaps in survey responses emerge even when comparing respondents within the same county.

A number of contemporaneous studies also measure partisan differences in responses to COVID19. ${ }^{1}$ Gadarian et al. (2020), Makridis and Rothwell (2020), and Wu and Huber (2020) show that

\footnotetext{
${ }^{1}$ Coverage in the media and some studies examine partisan heterogeneity in response to COVID-19 with no or few
} 
partisanship is a primary driver of attitudes about the pandemic and self-reported behaviors in surveys, and Druckman et al. (2020) show that affective polarization colors people's evaluations of the U.S. government response to the pandemic. Cornelson and Miloucheva (2020), Grossman et al. (2020), and Painter and Qiu (2020) demonstrate partisan differences in response to statelevel stay-at-home orders. Barrios and Hochberg (2020) show differences between Republican and Democratic areas in the frequency of COVID-related queries on Google and in movement patterns as measured in GPS data from a different source than the one we use. Fan et al. (2020) find differences in risk perceptions and social distancing in GPS and survey data across political parties and other demographics. Our results are broadly consistent with these other studies, but we believe that our paper contributes to the discussion through a unique combination of observational data analysis, survey work, and a theoretical model that helps understand the economic implications of our results.

Ash et al. (2020), Bursztyn et al. (2020) and Simonov et al. (2020) find that people social distance less if quasi-randomly exposed to news sources that argue that COVID-19 is less risky, suggesting that media exposure is one possible driver for our results. ${ }^{2}$

Our work contributes to a broader literature on what drives responses to pandemics (e.g., Blendon et al. 2008; Vaughan and Tinker 2009; Fineberg 2014). Mobilizing an effective public response to an emerging pandemic requires clear communication and trust (Holmes 2008; Taylor et al. 2009; van der Weerd et al. 2011; Vaughan and Tinker 2009). Risk perception, behavior changes, and trust in government information sources change as pandemics progress (Ibuka et al. 2010; Bults et al. 2011). Demographic characteristics, such as gender, income, geography, or social interactions, are important determinants of the adoption of recommended public health behaviors (Bish and Michie 2010; Ibuka et al. 2010; Bults et al. 2011; Chuang et al. 2015; Shultz et al. 2016; Gamma et al. 2017).

A related literature focuses on the consequences of political polarization for health behaviors (e.g., Iyengar et al. 2019 and Montoya-Williams and Fuentes-Afflick 2019). Party affiliation is correlated with physician recommendations on politicized health procedures, enrollment in government exchanges created under the Affordable Care Act, beliefs in the safety of vaccines, and hurricane evacuations (Hersh and Goldenberg 2016; Lerman et al. 2017; Sances and Clinton 2019;

controls for differential risk exposure or costs of social distancing (e.g., Economist 2020; Andersen 2020). Baker et al. (forthcoming) use transaction-level data and examine heterogeneity in consumption responses to COVID- 19 .

${ }^{2}$ Pastor and Veronesi (forthcoming) also find that Democrats are more risk averse than Republicans. Differences in risk aversion would not explain the differences in beliefs we find in Section 5, but are a possible complementary explanation for the observed partisan gap in social distancing. 
Trachtman 2019; Krupenkin 2018; Suryadevara et al. 2019; Long et al. 2019).

Finally, our work relates to broader literatures on partisan differences in trust and beliefs (e.g., Bartels 2002; Gaines et al. 2007) and adds to the increasing number of papers using GPS or related data to study social interactions (e.g., Dubé et al. 2017; Chen and Rohla 2018; Athey et al. 2019).

Sections 2, 3, 4, and 5, respectively, present our theoretical framework, data, GPS analysis, and survey results.

\section{Stylized Model}

In this section, we present a stylized model to clarify why it might matter if different types of people choose different amounts of social distancing. We embed an epidemiological model of disease transmission into an economic model with agents who maximize utility considering the expected private cost of disease. We consider how heterogeneity in perceived risks affects aggregate welfare.

\subsection{Epidemiological Model}

We use a discrete-time version of the standard SIR epidemiological model (Kermack and McKendrick 1927). In each period $t$, each person is in one of four states $\sigma \in\{S, I, R, D\}$, representing Susceptible, Infected, Recovered, and Deceased. The share of the population in each state at time $t$ is $s_{t}, i_{t}, r_{t}$, and $d_{t}$. Let $\beta$ represent disease infectiousness, and let $c_{t}$ denote an individual's amount of risky behavior at time $t$-for example, the amount of travel, dining out, failing to wash hands, and other activities that increase the risk of becoming Infected.

All people begin in the Susceptible state. A Susceptible person becomes Infected at time $t+1$ with probability $c_{t} \beta i_{t}$ and stays Susceptible with probability $\left(1-c_{t} \beta i_{t}\right)$. Infected people stay Infected for one period, after which they become Deceased with probability $\psi$ or Recovered with probability $(1-\psi)$. Both $D$ and $R$ are absorbing states.

Let $\theta$ index different types of people-for example, liberals and conservatives. Let $\omega_{\theta \sigma t}$ be a state variable representing the share of type $\theta$ that is in state $\sigma$ at time $t$. The population is of measure 1 , so $\sum_{\theta} \sum_{\sigma} \omega_{\theta \sigma t}=1$.

\subsection{Individual Decisions}

People of type $\theta$ earn flow utility $u_{\theta}\left(c_{t} ; \sigma_{t}\right)$, which depends on their risky behavior $c_{t}$ and their state $\sigma_{t}$. People discount the future at rate $\delta$ and maximize expected lifetime utility $\sum_{\tau=t}^{\infty} \delta^{\tau} u_{\theta}\left(c_{\tau} ; \sigma_{\tau}\right)$. 
Define $V_{\theta}(\sigma)$ as the expected lifetime utility of a person currently in state $\sigma$; note that this also implicitly depends on current and future population states $\omega_{\theta \sigma t}$. Being infected reduces utility, so we assume $V_{\theta}(S)>V_{\theta}(I)$ for any given current population state.

We focus on Susceptible people, as they comprise most of the population during the period we study and are the people who face a trade-off between the benefit of consumption and the risk of becoming infected. We can write their maximization problem as a Bellman equation, in which people maximize the sum of utility from risky behavior today and expected future utility:

$$
V_{\theta}\left(S_{t}\right)=\max _{c_{t}}\{\underbrace{u_{\theta}\left(c_{t} ; S_{t}\right)}_{\text {current utility from risky behavior }}+\underbrace{\delta\left[c_{t} \beta i_{t} V_{\theta}(I)+\left(1-c_{t} \beta i_{t}\right) V_{\theta}(S)\right]}_{\text {expected future utility }}\} .
$$

The first-order condition for privately optimal risky behavior is

$$
\underbrace{u_{\theta}^{\prime}}_{\text {marginal utility of risk }}=\underbrace{\beta i_{t}}_{\text {marginal infection probability private cost of infection }} \underbrace{\delta\left(V_{\theta}(S)-V_{\theta}(I)\right)}_{\text {. }} .
$$

The first-order condition shows that people choose their risky behavior to equate marginal benefit (more utility today) with private marginal cost (higher risk of infection, which reduces future utility). The equation illustrates that there are three reasons why risky behavior might vary across types. First is the marginal utility of risk (or equivalently, the marginal cost of social distancing): for example, people vary in how much they like travel and dining out, as well as in how easy it is to work from home. Second is the marginal infection probability: for example, local infection rate $i_{t}$ differs across geographic areas. Third is the private cost of infection: for example, infection is more harmful for people who are older or have underlying health conditions.

\subsection{Social Optimum}

It is difficult to know for sure whether people take too many or too few steps to reduce disease transmission during our study period. Thus, we do not consider the optimal consumption of $c$. Instead, we hold constant the total amount of risky behavior and ask whether the allocation across types is optimal. Tangibly, this means that we are not asking, "how much social distancing should people be doing?" Instead, we are asking, "holding constant the amount of social distancing people are doing, would some people ideally be doing less, and others ideally be doing more?"

Social welfare is the sum of utility across all people in all states: 


$$
W_{t}=\sum_{\theta} \sum_{\sigma} \omega_{\theta \sigma t} V_{\theta}\left(\sigma_{t}\right)
$$

Let $C_{t}$ denote the total risky behavior at time $t$ across all people. The (constrained) socially optimal outcome results from maximizing $W_{t}$ subject to the constraint that $C_{t}=\bar{C}_{t}$. Let $\lambda$ be the shadow price on that constraint; this reflects the loss from having too much or too little social distancing overall.

Consuming $c$ imposes two types of externalities. First, it imposes a positive pecuniary externality, as travel, dining out, and other risky activities help keep firms in business and workers employed. Second, it imposes a negative externality by increasing the person's infection probability, which increases the expected stock of infected people in the next period $\left(i_{t+1}\right)$ and then increases other Susceptible people's infection risk. Let $\phi_{t}$ denote the net externality per unit of consumption, which may be positive or negative; this becomes more negative as the contagion externality grows. We assume that these externalities are constant across people and that people do not account for the externalities when choosing $c_{t}^{*}$.

In the constrained social optimum, Susceptible people's consumption of $c_{t}$ would satisfy the following first-order condition:

$$
0=\underbrace{u_{\theta}^{\prime}-\beta i_{t} \delta\left(V_{\theta}(S)-V_{\theta}(I)\right)}_{\text {private marginal utility }}+\underbrace{\phi_{t}}_{\text {externality }}+\underbrace{\lambda}_{\text {shadow price }} .
$$

People who are not Susceptible do not account for transition risks. In the constrained social optimum, they set $0=u_{\theta}^{\prime}+\phi_{t}+\lambda$.

\subsection{Heterogeneous Risk Misperceptions}

We now allow people to misperceive risks. These misperceptions cause Susceptible people to choose too much or too little risky behavior relative to their private optimum, and heterogeneous misperceptions cause transfers across types and efficiency losses.

We now add $\theta$ subscripts to explicitly denote different parameters by type. Let $\mu_{t \theta}:=\beta i_{t} \delta\left(V_{\theta}(S)-V_{\theta}(I)\right)$ denote type $\theta$ 's expected utility cost due to infection from an additional unit of risky consumption. Let $\tilde{\mu}_{t \theta}$ denote type $\theta$ 's perception of that cost. Susceptible type $\theta$ consumers then set $c_{t \theta}$ according to the following modified first-order condition: 


$$
u_{\theta}^{\prime}=\tilde{\mu}_{t \theta}
$$

giving consumption denoted $c_{t \theta}^{*}$.

For illustrative purposes, imagine there are two types $\theta \in\{a, b\}$ in equal proportion, and that period $t$ marginal utility is linear and the same for both types, so $u_{\theta}^{\prime}(c)=u^{\prime}(c)$ for both types and $u^{\prime \prime}$ is a constant. Finally, without loss of generality, assume type $a$ perceives greater risk, so $\tilde{\mu}_{a \theta}>\tilde{\mu}_{b \theta}$. Our survey data show Democrats perceive greater risk, so one can think of Democrats as type $a$.

Define $\overline{\tilde{\mu}}_{t}:=\frac{1}{2}\left(\tilde{\mu}_{t a}+\tilde{\mu}_{t b}\right)$ as the average risk perception. With homogeneous risk perceptions, both types would set $c_{t}$ such that $u^{\prime}=\overline{\tilde{\mu}}_{t}$, giving homogeneous consumption denoted $\bar{c}_{t}$. With heterogeneous misperceptions, type $a$ consumes more and type $b$ consumes less; the consumption difference is:

$$
c_{t b}^{*}-c_{t a}^{*}=\frac{\tilde{\mu}_{t a}-\tilde{\mu}_{t b}}{-u^{\prime \prime}} .
$$

These consumption differences cause both transfers across types and efficiency losses.

Risk perceptions affect risky consumption, and risky consumption causes externalities, so the heterogeneous misperceptions cause transfers across groups. The net transfer from type $a$ to type $b$ from heterogeneous instead of homogeneous misperceptions is

$$
\underbrace{\frac{\tilde{\mu}_{t a}-\tilde{\mu}_{t b}}{-u^{\prime \prime}}}_{\text {consumption difference }} \cdot \underbrace{\phi_{t}}_{\text {externality }} .
$$

If $\phi_{t}>0$, i.e. the positive pecuniary externality from risky consumption outweighs the negative contagion externality, then heterogeneous misperceptions cause a net transfer from type $b$ to type $a$. Intuitively, we would say that Republicans are doing more to keep the economy going. On the other hand, if $\phi_{t}<0$, i.e. the negative contagion externality outweighs the positive pecuniary externality, then heterogeneous misperceptions cause a net transfer from type $a$ to type $b$. Intuitively, we would say that Democrats are doing more to reduce the spread of disease.

The efficiency cost in period $t$ from heterogeneous instead of homogeneous misperceptions are the two deadweight loss triangles around $\bar{c}_{t}$, with total area: 


$$
\Delta W_{t}=\frac{s_{t}}{2} \cdot \frac{(\overbrace{\tilde{\mu}_{t a}-\overline{\tilde{\mu}}_{t}}^{\text {misperception }})^{2}}{\underbrace{-u^{\prime \prime}}_{\text {slope of private marginal utility }}} .
$$

Intuitively, type $a$ people (Democrats) are doing too much social distancing, and type $b$ (Republicans) too little, relative to the (constrained) social optimum with homogeneous risk perceptions. Since the marginal cost of social distancing is increasing, society could achieve the same amount of social distancing at lower cost if type $a$ did less and type $b$ did more.

This model informs the empirical tests in the rest of the paper. In Sections 4 and 5 , we ask if Democrats and Republicans are reducing risk by different amounts. We use proxies to control for differences in actual risks and differences in the marginal costs of risk reduction-both of which could cause differential risk reduction to be socially optimal. In Section 5, we ask if Democrats and Republicans have different risk perceptions, which would generate the transfers and efficiency costs described above. In these analyses, we control for factors such as population density, health risks, and local coronavirus cases that could generate difference in actual risks across types. We also give a back-of-the-envelope estimate for the efficiency cost of heterogeneous misperceptions.

\section{Data}

\subsection{SafeGraph Mobile GPS Location Data}

Our analysis uses data from SafeGraph, aggregating GPS pings from about 45 million mobile devices and numerous applications to measure foot traffic patterns to a collection of points-ofinterest (POIs). POIs include retail shops, restaurants, movie theaters, hospitals, and many other public locations individuals may choose to go when leaving their house. For each POI, SafeGraph reports its geographic location, industry, and the total number of visitors in their mobile device panel that have visited each day.

Our primary analysis uses data from a period of 24 weeks, from January 27 to July 12, 2020. We aggregate visits across all POIs in a given county and week. We also separately aggregate visits by 2-digit NAICS code for each county and week. In a placebo analysis, we analyze data over earlier time periods (starting in January 2019).

We also use data from the SafeGraph Social Distancing data released as a part of their COVID- 
19 response. This data is available since January 1, 2019 and updated regularly. We use data over the same 24 week period. This data contains alternative measures of social distancing beyond POI visits, such as the number of devices leaving their assigned geohash-7 home, the number of other census block groups visited, or the median time spent away from home across devices.

We supplement the SafeGraph data with various other sources of county and census block group data. For demographic information on age, race, education, income, occupation, and poverty status at the county-level, we aggregate census block group data from SafeGraph Open Census to the county level. ${ }^{3}$ We add weather statistics on temperature and precipitation from gridMET (Abatzoglou 2011), aggregated to the county-level. ${ }^{4}$ For each county, we define county partisanship to be the proportion of total votes received by President Donald Trump in the 2016 election (MIT Election Data and Science Lab 2018). We use county-level data on COVID-19 cases and deaths from The New York Times (2020). We also add data on county or state stay-at-home policies from a variety of sources (as in Allcott et al. 2020). ${ }^{5}$

\subsection{Survey}

To supplement these data, we ran an online survey with a sample of American adults to study partisan gaps in beliefs about and responses to COVID-19 at the individual level. The survey was conducted from April 4-7, 2020 with Prime Panels from CloudResearch, a market research firm with access to 50 million participants. We recruited 2,000 participants to complete the study. Participants are broadly representative of U.S. adults in terms of party affiliation, age, gender, and race. In addition, we weighted observations so that age, gender, and race distributions match 2010 Census data and party affiliation matches recent Gallup polling data (Gallup 2020).

Participants were asked for their party affiliation on a seven-point scale, ranging from "Strongly Democratic" to "Strongly Republican." We transform the seven-point party affiliation scale to range between 0 (Strongly Democratic) and 1 (Strongly Republican), with intermediate values equally spaced.

The survey asked for demographic information (zip code, age, race, gender, income, education, number of children, and health characteristics). It then asked about news consumption habits and

\footnotetext{
${ }^{3}$ The SafeGraph Open Census data is derived from the 2016 5-year ACS at the census block group level.

${ }^{4}$ We thank Jude Bayham for sharing aggregated versions of this dataset with the SafeGraph COVID-19 response community, originally constructed for Burkhardt et al. (2019).

${ }^{5}$ We combine policy data from: Keystone Strategy; a crowdsourcing effort from Stanford University and the University of Virginia; Hikma Health; and The New York Times.
} 
trust before and during COVID-19. Then, there were several questions about social distancing: self-reported social distancing in response to COVID-19, beliefs about the risk of not distancing, and the appropriate trade-off between going out more to help the economy versus going out less to avoid spreading COVID-19.

We next elicited beliefs about the number of new COVID-19 cases that would be confirmed in the US in April, 2020, with 1,013 subjects (51 percent) being financially incentivized for predic-

tions that are closer to the correct answer. ${ }^{6}$ The remaining 987 (49 percent) of subjects were not incentivized.

The primary four outcome variables are participants' answers to the three social-distancing questions and the one prediction question. These analyses correspond to the "main analyses" in our pre-analysis plan (AEA RCT Registry 5632). In the interest of space, we do not discuss the plan's "exploratory analyses" in this paper.

\section{SafeGraph Empirical Specification and Results}

Figure 2 presents geographic variation in social distancing, partisanship, COVID-19 incidence, and stay-at-home orders. Panels A and B illustrate a strong geographic correlation between the counties with weaker social distancing responses during the week of peak social distancing (April 6-12, 2020) and those with higher Republican vote shares. However, partisanship is also strongly correlated with COVID-19 incidence (Panel C) and earlier stay-at-home orders (Panel D).

Figure 3 reports trends in social distancing and COVID-19 incidence separately for Republican and Democratic counties. Panel A shows that the overall number of POI visits was relatively constant until COVID-19 cases begin emerging in the United States in March. Mobility levels then fell until reaching a minimum during the week of April 6-12, 2020, followed by a gradual recovery that remained below pre-pandemic levels as of July 6-12, 2020. Throughout this pandemic period, Democratic counties exhibited a larger drop in weekly POI visits than their Republican counterparts with this partisan gap generally growing over time. However, as Panel B demonstrates, Democratic counties also exhibited a larger rise in COVID-19 cases and deaths. Appendix Figure A1 shows that, over the same time period in 2019, POI visits displayed a noticeable but smaller partisan gap.

${ }^{6}$ Participants who made financially incentivized predictions were told that we would randomly select 10 participants who would receive a payment of $(\$ 100-\Delta)$, where $\Delta$ is the percentage point difference between their answer and the true value. 
Our main empirical specification takes the following form

$$
\log \left(c_{i t}\right)=\alpha_{t} \rho_{i}+\mu_{i}+\gamma_{t}+X_{i t} \cdot \gamma_{t}+\varepsilon_{i t}
$$

where $c_{i t}$ is the number of POI visits in county $i$ during week $t, \alpha_{t}$ are the time-varying coefficients on county partisanship $\rho_{i}, \mu_{i}$ and $\gamma_{t}$ represent county and week fixed effects respectively, $X_{i t}$ are non-parametric and time-varying controls, and $\varepsilon_{i t}$ is the county-specific error term. ${ }^{7}$ We chose our covariates $X_{i t}$ to flexibly control for the four channels of divergent behavior highlighted in equation (2). Standard errors are clustered at the state-level throughout unless specified otherwise.

Figure 4 reports our estimates of $\alpha_{t}$ under various sets of covariates chosen to incrementally control for the mechanisms highlighted by our model.

In Panel A, we only include county and time fixed effects. This measures the extent to which these two groups' behavior diverges with the rise of COVID-19 via any of the aforementioned channels. Throughout February, there are no significant partisan differences in POI visits relative to the January 27 week baseline. However, as COVID-19 begins to emerge in the United States, partisan differences arise and grow throughout the weeks of March and persist at least through early July.

These results do not control for differences in public policies, which themselves may be a function of the partisan leanings of government officials. In Panel B, adding state-time fixed effects to control for state-level policies in response to COVID-19 along with other state-level temporal shocks causes the partisan differences to attenuate only slightly. ${ }^{8}$

In Panel C, we flexibly control for various health, ${ }^{9}$ economic, ${ }^{10}$ and weather ${ }^{11}$ characteristics of the county. We view the health controls as proxies for the marginal infection probability and the private cost of infection, and we view the economic controls as proxies for the marginal cost of social distancing, though each group of controls could proxy for other factors as well. We

\footnotetext{
${ }^{7}$ We normalize $\alpha_{t}$ relative to the first week.

${ }^{8}$ State-time fixed effects also control for the partisan alignment between the governor and the state population, which may impact responses to social distancing orders (see, e.g., Painter and Qiu 2020).

${ }^{9}$ Health controls include: an indicator for whether a county has been under a stay-at-home order; log of one plus the number of confirmed COVID-19 cases in the county; log of one plus the number of COVID-19 deaths in the county; $\log$ of one plus the county population density (individuals per square kilometer); and share of the population age 65+.

${ }^{10}$ Economic controls include: share of the population with at least a bachelor's degree; share in poverty; share with household income $\geq \$ 100,000$; shares white, black, and asian; share commuting by public transportation; share currently enrolled in undergraduate study; and shares of occupations in various categories (management, business, science, and art; services; sales and office occupations; natural resources, construction, and maintenance).

${ }^{11}$ Weather controls include daily high temperature, daily low temperature, and amount of precipitation averaged across days within a week.
} 
include these controls nonparametrically via indicators for decile bins within a week, which we interact with time fixed effects in order to allow the coefficients on these indicators to vary flexibly across time. Although these controls attenuate partisan differences, they remain economically and statistically significant. Appendix Figure A2 shows that these strong partisan differences do not appear over the same time period in 2019 conditional on the same controls. These results are consistent with behavioral differences driven by partisan misperceptions of risks at the group-level.

To better understand the magnitudes of this partisan gap, we compare the difference between very Republican and very Democratic counties to contemporaneous mobility levels and to overall social distancing relative to January. The estimate of our partisan gap coefficient $\alpha_{t}$ is 0.292 by the week starting April 6 (the week with the fewest number of visits) and 0.386 in the week starting May 11 (the week with the largest partisan gap). These estimates imply that going from a county with the 10th to the 90th percentile in Republican vote share is associated with 11.5 and 15.2 percent increases in the number of POI visits during these two weeks respectively. ${ }^{12}$ The 11.5 percent gap during the week of April 6 corresponds to 6.5 percent of the total change in POI visits between the weeks of January 27 and April 6, and the partisan gap during the week of May 11 is comparable in size to 15.0 percent of the total change in POI visits between the weeks of January 27 and May 11. ${ }^{13}$ The partisan gap in social distancing between very Republican and very Democratic counties is economically meaningful but only accounts for a limited portion of overall social distancing.

In Appendix Figure A3, we report sensitivity to various alternative specifications. Panels A and B use alternative sets of controls. Panel C replaces the measure of partisanship with a discrete indicator for certain quantiles of the Republican vote share distribution. Panel D drops counties that are very small (less than 3,000 people), very large (greater than 500,000 people), or are in states with early COVID-19 outbreaks (California, Washington, and New York). Panel E restricts the sample to counties from certain portions of the Republican vote share distribution. Panel F weights observations by the county's population, uses standard errors clustered at the county-level, and examines sensitivity to the start date. Except when restricting to counties in the top half of the Republican vote share, none of the alternative specifications change the central conclusion

\footnotetext{
${ }^{12}$ The difference between the 90th and 10th percentile of Republican vote share is $0.807-0.413=0.394$.

${ }^{13}$ Between the weeks of January 27 and April 6 (May 11), POI visits decreased by 64.0 (50.3) percent. We compare

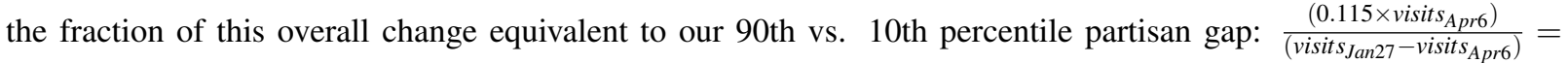
$0.115 \times \frac{\left(\text { visits }_{\text {Apr }} / \text { visits }_{\text {Jan } 27}\right)}{\left(\text { visits Jan27 }^{- \text {visits }} \text { Apr } 6\right) / \text { visits Jan } 27}=0.115 \times \frac{0.360}{0.640}=0.065$. The fraction of social distancing during the week of May 11 is similarly derived.
} 
regarding partisan differences in social distancing in March through at least early July.

Appendix Figure A4 aggregates the number of POI visits at the electoral precinct level and shows similar partisan gaps, even when including county-time fixed effects. Again, these patterns are not present in 2019 (Appendix Figure A5). Precinct-level analysis faces several limitations that lead us to prefer our county-level specification. ${ }^{14}$

Appendix Figure A6 examines heterogeneity across industries by aggregating POI visits to the county level after restricting to certain 2-digit NAICS codes. Consistent with the narrative around COVID-19, we see the strongest partisan differences emerge with POIs in the accommodations and food, entertainment, and retail industries. The partisan differences in visits to health care POIs are generally smaller and are statistically significant in fewer weeks.

Appendix Figure A7repeats Panel C of Figure 4, but uses POI visits aggregated at the day level. The partisan differences emerge for both weekdays and weekends, suggesting these differences are not driven solely by differences in work-from-home policies.

Appendix Figure A8 considers alternative measures of social distancing derived from SafeGraph's Social Distancing data. Statistically significant partisan differences emerge in March through at least early July for the log number of devices leaving home, the log number of stops made in non-home census block groups, the log of the median time away from home (Panel A), and the share of devices leaving home (Panel B). ${ }^{15}$ In Panel C, we conduct our alternative social distancing analysis at the precinct level while including county-time fixed effects. For the log number of devices leaving home and the log number of stops made in non-home census block groups, we find an economically and statistically significant partisan gap emerge starting in April and persisting through early July (though see footnote 14 for limitations of the precinct-level analysis).

\footnotetext{
${ }^{14}$ We note several limitations of our precinct-level analysis. Due to the limited availability of 2016 precinct-level shapefiles, our precinct-level analysis includes only 42 states (see Appendix A.1.2). Partisanship is measured at the precinct-level, while social distancing and our health, weather, and economic controls are generally measured at the census block group level. The latter set of variables are then mapped to precincts based on geographic overlap using the procedure described in Appendix A.1.2, potentially introducing correlated measurement error between our outcome and non-partisanship controls. Finally, POI visits are allocated to geographies by merchant location whereas partisanship is measured among residents. With smaller geographies, it becomes increasingly likely that visitors to a POI come from a different home geography, resulting in mismatch between visits and partisanship.

${ }^{15}$ A key issue with the SafeGraph social distancing data is sample attrition. SafeGraph restricts the panel to devices with observed location pings in a given time period. For some applications, the frequency of location pings depends on device mobility. If devices are immobile at home or turned off, they may not generate location pings and would then be dropped from the sample. The total number of active devices changes over our sample period in a manner consistent with sample attrition. Given these issues, we prefer measures of social distancing derived solely from external activity (e.g., POI visits) that do not contain the same measurement error problems. We attempt to correct for the differential attrition in our measure of the share of devices leaving home (see Appendix Figure A8 footnotes for correction).
} 


\section{Survey Results}

Turning to the results of our survey, we first confirm that individuals' beliefs related to COVID-19 are strongly associated with their social distancing behaviors. We find that a one standard deviation (SD) increase in beliefs about the efficacy of social distancing, as described below, is associated with a 0.323 SD increase in self-reported social distancing (SE 0.022; p $<0.001$ ), controlling for demographic characteristics and state fixed effects. Similarly, a one SD increase in beliefs about the number of future cases in the US is associated with a 0.066 SD increase in self-reported social distancing (SE 0.023; p = 0.004).

Next, we show that there exist individual-level partisan differences in (self-reported) social distancing behaviors and attitudes, consistent with the GPS analysis presented above. We then show that beliefs about the effectiveness of social distancing and predictions of the spread of COVID-19 follow the same partisan patterns. Our main empirical specification regresses normalized responses on party:

$$
y_{i}=\kappa+\alpha \rho_{i}+\gamma X_{i}+\varepsilon_{i}
$$

where $y_{i}$ is the number of standard deviations above the mean for response $i, \rho_{i}$ is the continuous measure of Republican party lean from 0 to $1, X_{i}$ are demographic and location controls, and $\varepsilon_{i}$ is an error term.

Figure 5 shows consistent evidence for partisan differences in social distancing, both with and without control variables. ${ }^{16}$ On average, participants report reducing contact by 70.0 percent, with a SD of 24.5 percent. After including controls, strong Democrats report engaging in 0.18 SD more contact reduction than strong Republicans. This corresponds to a gap in contact reduction of 72.1 percent for strong Democrats versus 67.8 percent for strong Republicans. Similarly, Democrats find it significantly more important to stay inside to prevent the spread of the virus versus go outside to help the economy, and the difference between strong partisans is 0.23 SD.

We then examine the partisan differences in underlying beliefs regarding COVID-19 severity and efficacy of social distancing. We find that Democrats' belief regarding the probability of catching COVID-19 without any social distancing is higher than the analogous belief held by Republicans. On average, participants assess this probability to be 55.0 percent (SD 31.9 percent).

\footnotetext{
${ }^{16}$ These differences are also present when we do not weight observations for national representativeness, as shown in Appendix Figure A9. For detail on observation weights, see Appendix A.2.1.
} 
Strong Democrats believe this probability is 60.5 percent, which is 0.34 SD larger than the 49.6 percent belief held by strong Republicans.

We next consider beliefs about future COVID-19 cases in the entire US. We tell participants the number of cases by March 31 and ask them to predict the number of cases in April. On average, participants predict 202,810 new cases in April 2020 (SD 233,343 cases, due to a long right tail). ${ }^{17}$ Strong Democrats predict 231,129 future cases on average, which is 0.24 SD more than the 174,491 predicted by strong Republicans. ${ }^{18}$ Bullock et al. (2015) and Prior et al. (2015) show that partisan differences on factual questions often shrink under incentives due to "partisan cheerleading" rather than differences in true beliefs. When we randomize whether subjects' predictions are incentivized for accuracy, we do not find evidence that the partisan gap decreases. ${ }^{19}$ This supports the view that Democrats and Republicans genuinely differ in their beliefs about the severity of COVID-19. Appendix Figure A10 shows that on an explicitly political question, incentives do significantly reduce the partisan gap, consistent with previous findings.

Appendix Figure A11 shows that comparing individuals within the same county produces qualitatively similar results, complementing the county-level partisan gap observed in the GPS analysis. However, since 21.5 percent of participants are the only participant from their county, statistical precision is lower and low-population counties are underweighted.

Finally, we do a back-of-the-envelope estimation of the deadweight loss from equation (8). We assume that agents have the same quadratic flow utility functions $u(c)=\frac{v}{2} c^{2}+\eta c+k$ and normalize parameters so that $c^{*}=1$ is the amount of risky behavior chosen in the absence of the coronavirus. (Formally, if $\beta=0$, all agents choose $c^{*}(0)=1$, i.e., $v=-\eta \leq 0$.) We then consider what happens when partisan perceptions differ about infectiousness $\beta$. From our survey, we find that the median participant's willingness-to-accept for "cutting off all in-person contact with people outside your household for one month" versus "following your normal routine" (i.e., one month of $c=0$ instead of $c=1$ ) is $\$ 1500$. From the survey data above, we approximate that Democrats reduce consumption by 72.1 percent and Republicans reduce by 67.8 percent. This difference implies that, after controlling for observables that measure private costs and benefits of social distancing, Democrats perceive that the expected utility loss from following normal routines

\footnotetext{
${ }^{17}$ These averages are calculated after winsorizing at the 5-percent level to account for outliers.

${ }^{18}$ The actual number of confirmed April COVID cases was 901,670 (https://www.worldometers.info/coronavirus/country/us/). Subjects' underprediction might be due to misunderstanding of exponential growth, generic overoptimism, anchoring $(177,226$ was given as the reference number for cases by March 31), or to some other factor.

${ }^{19}$ The gap slightly increases, though the effect is statistically insignificant. Regressing predictions on the interaction between party and incentives corresponds to the specification in our pre-analysis plan.
} 
instead of cutting off all contact is $\tilde{\mu}_{t R}-\tilde{\mu}_{t D}=\$ 129$ per month higher than what Republicans perceive. $^{20}$

Plugging the perceived utility loss estimates into equation (8), we compare the deadweight loss if partisans have different perceived risks $\left(\mu_{t D}^{*}, \mu_{t R}^{*}\right)$ compared to if they have the same perceived risk $\left(\mu_{t D}^{*}+\mu_{t R}^{*}\right) / 2$. Using an estimate of 330 million people in the US and 99 percent of the country being susceptible, we estimate that partisan differences in risk misperceptions generate a deadweight loss of approximately $\Delta W=\$ 8.24$ per person per year, or $\$ 2.7$ billion for the US per year.

\section{Conclusion}

If Republicans and Democrats disagree about the potential risks, they may also differ in how much they reduce the risk of disease transmission through social distancing and other actions. In this case, our model shows how society ends up with more disease transmission at higher economic cost than if people had the same beliefs.

Our empirical results show that partisan gaps in beliefs and behavior are real. GPS evidence reveals significant partisan gaps in actual social distancing behaviors. Survey evidence shows substantial gaps between Republicans and Democrats in beliefs about the severity of COVID19 and the importance of social distancing. The raw partisan differences partly reflect the fact that Democrats are more likely to live in the dense, urban areas hardest hit by the crisis, and to be subject to policy restrictions - in other words, to face stronger individual incentives for social distancing. Even after controlling carefully for such factors, however, the partisan gaps remain statistically and economically significant.

One explanation for these results is that media sources have sent divergent messages about the coronavirus. Appendix Figure A12 shows that the partisan gaps in the survey data are smaller when the partisanship of news consumption is controlled for, and that news partisanship is statistically significantly correlated with beliefs even when party is controlled for. While our evidence does not permit us to pin down the ultimate causes of partisan divergence, these patterns are consistent with divergent messaging playing an important role in driving differences in beliefs and behavior.

\footnotetext{
${ }^{20}$ We use the willingness-to-accept data to say that $u(1)-u(0)=\frac{1}{2} \eta=\$ 1500$, so that $\eta=\$ 3000$. Then, using that Republicans choose consumption $c_{t R}^{*}=1-0.678=0.322$ and Democrats choose $c_{t D}^{*}=1-0.721=0.279$, we have from equation 6 that $0.322-0.279=\frac{\tilde{\mu}_{t R}-\tilde{\mu}_{t D}}{\$ 3000}$, so that $\tilde{\mu}_{t R}-\tilde{\mu}_{t D}=\$ 129$.
} 


\section{References}

Abatzoglou, John T. 2011. Development of Gridded Surface Meteorological Data for Ecological Applications and Modelling. International Journal of Climatology. http://www.climatologylab.org/gridmet.html. Aleem, Zeeshan. 2020. A New Poll Shows a Startling Partisan Divide on the Dangers of the Coronavirus. Vox. March 15. https://www.vox.com/2020/3/15/21180506/coronavirus-poll-democrats-republicanstrump.

Allcott, Hunt, Levi Boxell, Jacob Conway, Billy Ferguson, Matthew Gentzkow, and Benny Goldman. 2020. The Effect of Stay-at-Home Orders on Mobility, Economic, and Health Outcomes during the Coronavirus Pandemic. Working Paper.

Andersen, Martin. 2020. Early Evidence on Social Distancing in Response to COVID-19 in the United States. Working Paper.

Ash, Elliott, Sergio Galletta, Dominik Hangartner, Yotam Margalit, and Matteo Pinna. 2020. The Effect of Fox News on Health Behavior During COVID-19. Working Paper.

Athey, Susan, Billy Ferguson, Matthew Gentzkow, and Tobias Schmidt, 2019. Experienced Segregation. Working Paper.

Athey, Susan, Keystone Strategy, and Marco Iansiti. 2020. Coronavirus City and County Non-Pharmaceutical Intervention Rollout Date Dataset. https://www.keystonestrategy.com//coronavirus-covid19-interventiondataset-model/. Accessed on May 7, 2020.

Baker, Scott R., R.A. Farrokhnia, Steffen Meyer, Michaela Pagel, Constantine Yannelis. Forthcoming. How Does Household Spending Respond to an Epidemic? Consumption During the COVID-19 Pandemic. Review of Asset Pricing Studies.

Barrios, John M. and Yael V. Hochberg. 2020.Risk Perception Through the Lens of Politics in the Time of the COVID-19 Pandemic. Working Paper.

Bartels, Larry M. 2002. Beyond the Running Tally: Partisan Bias in Political Perceptions. Political Behavior. 24(2): 117-150.

Beauchamp, Zack. 2020. The Stunning Contrast Between Biden and Trump on Coronavirus. Vox.com. https://www.vox.com/policy-and-politics/2020/3/12/21177135/coronavirus-covid-19-pandemic-trumpbiden-speeches

Bish, Alison, and Susan Michie. 2010. Demographic and Attitudinal Determinants of Protective Behaviours During a Pandemic: A Review. British Journal of Health Psychology. 15(4): 797-824.

Blendon, Robert J., Lisa M. Koonin, John M. Benson, Martin S. Cetron, William E. Pollard, Elizabeth W. Mitchell, Kathleen J. Weldon, and Melissa J. Herrmann. 2008. Public Response to Community Mitigation Measures for Pandemic Influenza. Emerging Infectious Diseases. 14(5): 778.

Bullock, John G., Alan S. Gerber, Seth J. Hill, and Gregory A. Huber. 2015. Partisan Bias in Factual Beliefs About Politics. Quarterly Journal of Political Science. 10(4): 519-578.

Bults, Marloes, Desirée JMA Beaujean, Onno de Zwart, Gerjo Kok, Pepijn van Empelen, Jim E van Steenbergen, Jan Hendrik Richardus, and Hélène ACM Voeten. 2011. Perceived Risk, Anxiety, and Behavioural Responses of the General Public During the Early Phase of the Influenza A (H1N1) Pan- 
demic in the Netherlands: Results of Three Consecutive Online Surveys. BMC Public Health. 11(1): 2.

Burkhardt, Jesse, Jude Bayham, Ander Wilson, Ellison Carter, Jesse D. Berman, Katelyn O’Dell, Bonne Ford, Emily V. Fischer, and Jeffrey R. Pierce. 2019. The Effect of Pollution on Crime: Evidence From Data on Particulate Matter and Ozone. Journal of Environmental Economics and Management 98: 102267.

Bursztyn, Leonardo, Aakaash Rao, Christopher Roth, and David Yanagizawa-Drott. 2020. Misinformation During a Pandemic. Working Paper.

Chen, M. Keith, and Ryne Rohla. 2018. Politics Gets Personal: Effects of Political Partisanship and Advertising on Family Ties. Science. 360: 1020-1024.

Chuang, Ying-Chih, Ya-Li Huang, Kuo-Chien Tseng, Chia-Hsin Yen, and Lin-hui Yang. 2015. Social Capital and Health-Protective Behavior Intentions in an Influenza Pandemic. PloS One. 10(4).

Coppins, McKay. 2020. Trump's Dangerously Effective Coronavirus Propaganda. TheAtlantic.com. https://www.theatlantic.com/politics/archive/2020/03/trump-coronavirus-threat/607825/.

Cornelson, Kirsten, and Boriana Miloucheva. 2020. Political Polarization, Social Fragmentation, and Cooperation During a Pandemic. Working Paper.

Druckman, James, Samara Klar, Yanna Krupnikov, Matthew Levendusky, and John Barry Ryan. 2020. How Affective Polarization Shapes Americans' Political Beliefs: A Study of Response to the COVID-19 Pandemic. Working Paper.

Dubé, Jean-Pierre, Zheng Fang, Nathan Fong, and Xueming Luo. 2017.Competitive Price Targeting with Smartphone Coupons. Marketing Science. 36(6): 944-975.

Economist. 2020. Democrats Seem to Take Social Distancing More Seriously Than Republicans. Economist.com April 4. https://www.economist.com/united-states/2020/04/04/democrats-seem-to-take-social-distancingmore-seriously-than-republicans

Fan, Ying, A. Yesim Orhun, and Dana Turjeman. 2020. Heterogeneous Actions, Beliefs, Constraints and Risk Tolerance During the COVID-19 Pandemic. Working Paper.

Fineberg, Harvey V. 2014. Pandemic Preparedness and Response-Lessons from the H1N1 Influenza of 2009. New England Journal of Medicine. 370(14), 1335-1342.

Gadarian, Shana Kushner, Sara Wallace Goodman, and Thomas B. Pepinsky. 2020. Partisanship, Health Behavior, and Policy Attitudes in the Early Stages of the COVID-19 Pandemic. Working Paper.

Gaines, Brian J., James H. Kuklinski, Paul J. Quirk, Buddy Peyton, and Jay Verkuilen. 2007. Same Facts, Different Interpretations: Partisan Motivation and Opinion on Iraq. Journal of Politics. 69(4): 957974.

Gallup. 2020. Party Affiliation. Gallup. https://news.gallup.com/poll/15370/party-affiliation.aspx

Gamma, Anna E., Jurgita Slekiene, Gregor von Medeazza, Fredrik Asplund, Placido Cardoso, and HansJoachim Mosler. 2017. Contextual and Psychosocial Factors Predicting Ebola Prevention Behaviours Using the Ranas Approach to Behaviour Change in Guinea-Bissau. BMC Public Health. 17(1): 446. 
Grossman, Guy, Soojong Kim, Jonah Rexer, and Harsha Thirumurthy. 2020. Political Partisanship Influences Behavioral Responses to Governors' Recommendations for COVID-19 Prevention in the United States. Working Paper.

Hersh, Eitan D. and Matthew N Goldenberg. 2016. Democratic and Republican Physicians Provide Different Care on Politicized Health Issues. Proceedings of the National Academy of Sciences. 113(42): 11811-11816.

Holmes, Bev J. 2008. Communicating About Emerging Infectious Disease: The Importance of Research. Health, Risk \& Society. 10(4): 349-360.

Ibuka, Yoko, Gretchen B. Chapman, Lauren A. Meyers, Meng Li, and Alison P. Galvani. 2010. The Dynamics of Risk Perceptions and Precautionary Behavior in Response to 2009 (H1N1) Pandemic Influenza. BMC Infectious Diseases. 10(1): 296.

Iyengar, Shanto, Yphtach Lelkes, Matthew Levendusky, Neil Malhotra, and Sean J. Westwood. 2019. The Origins and Consequences of Affective Polarization in the United States. Annual Review of Political Science. 22: 129-146.

Kantrowitz, Alex. 2020. Conservative Media Still Isn't Sure What to Think About the Coronavirus. Buzzfeed News. March 18. https://www.buzzfeednews.com/article/alexkantrowitz/conservative-mediastill-isnt-sure-coronavirus

Kermack, William Ogilvy, and Anderson G. McKendrick. 1927. A Contribution to the Mathematical Theory of Epidemics. Proceedings of the Royal Society of London. Series A, Containing Papers of a Mathematical and Physical Character. 115(772): 700-721.

Krupenkin, Masha. 2018. Does Partisanship Affect Compliance With Government Recommendations? Political Behavior 1.

Lerman, Amy E., Meredith L. Sadin, and Samuel Trachtman. 2017. Policy Uptake as Political Behavior: Evidence From the Affordable Care Act. American Political Science Review. 111(4): 755-770.

Long, Elisa, M. Keith Chen, and Ryne Rohla. 2019. Political Storms: Emergent Partisan Skepticism of Hurricane Risks. Working Paper.

Makridis, Christos, and Jonathan T. Rothwell. 2020. The Real Cost of Political Polarization: Evidence from the COVID-19 Pandemic. Working Paper.

Marist. 2020. March 13th and 14th Survey of American Adults. http://maristpoll.marist.edu/wp-content/uploads/2020/03/NPR_PBS-NewsHour_Marist-Poll_USA-NOSand-Tables_2003151338.pdf.

McCarthy, Tom. 2020. Disunited States of America: Responses to Coronavirus Shaped by Hyper-Partisan Politics. The Guardian. Mar 29. https://www.theguardian.com/us-news/2020/mar/29/america-statescoronavirus-red-blue-different-approaches

Mervosh, Sarah, Denise Lu, and Vanessa Swales. 2020. See Which States and Cities Have Told Residents to Stay at Home. The New York Times. https://www.nytimes.com/interactive/2020/us/coronavirus-stay-at-home-order.html. Accessed on May 7, 2020 via Wayback Machine. 
MIT Election Data and Science Lab. 2018. County Presidential Election Returns 2000-2016. Harvard Dataverse. V42. https://doi.org/10.7910/DVN/NH5S2I

Montoya-Williams, Diana and Elena Fuentes-Afflick. 2019. Political Determinants of Population Health. JAMA Network Open. 2(7): e197063.

Noah, Cray, Senan Ebrahim, Henry Ashworth, Ali Ebrahim, Adesh Kadambi, Tara Pattilachan, Dani Kiyasseh, Melecia Wright, Eliza Nguyen, and Hassaan Ebrahim. 2020. COVID-19 US County Policies, Hikma Health. https://github.com/hikmahealth/covid19countymap. Accessed on May 7, 2020.

Painter, Marcus, and Tian Qiu. 2020. Political Beliefs Affect Compliance with COVID-19 Social Distancing Orders. Working Paper.

Pastor, Lubos and Pietro Veronesi. Forthcoming. Political Cycles and Stock Returns. Journal of Political Economy.

Piacenza, Joanna. 2020. Tracking Public Opinion on the Coronavirus. Morning Consult. https://morningconsult.com/form/tracking-public-opinion-on-the-coronavirus/.

Prior, Markus, Gaurav Sood, and Kabir Khanna. 2015. You Cannot Be Serious: The Impact of Accuracy Incentives on Partisan Bias in Reports of Economic Perceptions. Quarterly Journal of Political Science. 10(4): 489-518.

Ritchie, Jacob, Tum Chaturapruek, Mark Whiting, J.D. Zamfirescu-Pereira, Mitchell Gordon, Jackie Yang, Tianshi Li, Amy Zhang, Catherine Mullings, Rajan Vaish, Golrokh Emami, Danae Metaxa, Mandy Wilson, Achla Marathe, Stephen Eubank, Madhav Marathe, and Michael Bernstein. Crowdsourced COVID-19 Intervention Data. https://socialdistancing.stanford.edu/. Accessed on May 7, 2020.

Saad, Lydia. 2020. Americans Step up Their Social Distancing Even Further. Gallup. https://news.gallup.com/opinion/gallup/298310/americans-step-social-distancing-even-further.aspx.

SafeGraph. 2020. https://docs.safegraph.com/docs

Sances, Michael W. and Joshua D. Clinton. 2019. Who Participated in the ACA? Gains in Insurance Coverage by Political Partisanship. Journal of Health Politics, Policy and Law. 44(3): 349-379.

Shultz, James M., Janice L. Cooper, Florence Baingana, Maria A. Oquendo, Zelde Espinel, Benjamin M. Althouse, Louis Herns Marcelin et al. 2016. The Role of Fear-Related Behaviors in the 2013-2016 West Africa Ebola Virus Disease Outbreak. Current Psychiatry Reports. 18(11): 104.

Simonov, Andrey, Szymon Sacher, Jean-Pierre Dubé, and Shirsho Biswas. 2020. The Persuasive Effect of Fox News: Non-Compliance with Social Distancing During the Covid-19 Pandemic. Working Paper.

Stanley-Becker, Isaac and Chelsea Janes. 2020. As Virus Takes Hold, Resistance to Stay-At-Home Orders Remains Widespread - Exposing Political and Social Rifts. Washington Post. April 2. https://www.washingtonpost.com/politics/as-virus-takes-hold-resistance-to-stay-at-home-orders-remainswidespread-exposing-political-and-social-rifts/2020/04/02/d87314e0-7436-11ea-85cb-8670579b863d_story.html

Suryadevara, Manika, Cynthia A. Bonville, Donald A. Cibula, Joseph B. Domachowske, and Amar C. Suryadevara. 2019. Associations Between Population Based Voting Trends During the 2016 US Presidential Election and Adolescent Vaccination Rates. Vaccine. 37(9): 1160-1167. 
Taylor, Melanie, Beverley Raphael, Margo Barr, Kingsley Agho, Garry Stevens, and Louisa Jorm. 2009. Public Health Measures During an Anticipated Influenza Pandemic: Factors Influencing Willingness to Comply. Risk Management and Healthcare Policy. 2: 9.

The New York Times. 2020. Coronavirus (Covid-19) Data in the United States. https://github.com/nytimes/covid19-data Accessed on May 12, 2020.

Trachtman, Samuel. 2019. Polarization, Participation, and Premiums: How Political Behavior Helps Explain Where the ACA Works, and Where It Doesn't. Journal of Health Politics, Policy and Law. 44(6): 855-884.

van der Weerd, Willemien, Daniëlle RM Timmermans, Desirée JMA Beaujean, Jurriaan Oudhoff, and Jim E. van Steenbergen. 2011.Monitoring the Level of Government Trust, Risk Perception and Intention of the General Public to Adopt Protective Measures During the Influenza A (H1N1) Pandemic in the Netherlands. BMC Public Health. 11(1): 575.

Vaughan, Elaine, and Timothy Tinker. 2009. Effective Health Risk Communication About Pandemic Influenza for Vulnerable Populations. American Journal of Public Health. 99(S2): S324-S332.

Voting and Election Science Team. 2018. 2016 Precinct-Level Election Results. Harvard Dataverse. V37. https://doi.org/10.7910/DVN/NH5S2I

Wu, Jennifer D. and Gregory A. Huber. 2020. Partisanship Differences in Social Distancing May Originate in Norms and Beliefs: Results from Novel Data. Working Paper. 
Figure 1: Partisan Differences in Perceived Risk and Social Distancing

Panel A: Concern over Spread of Coronavirus

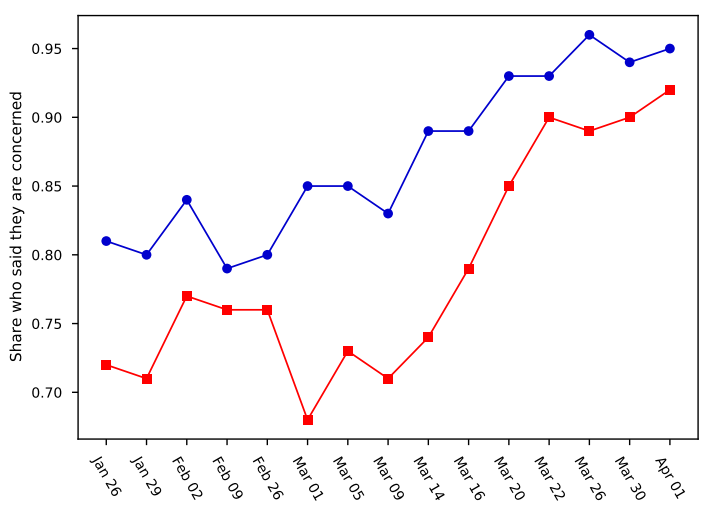

Panel C: Share Avoiding Public Places

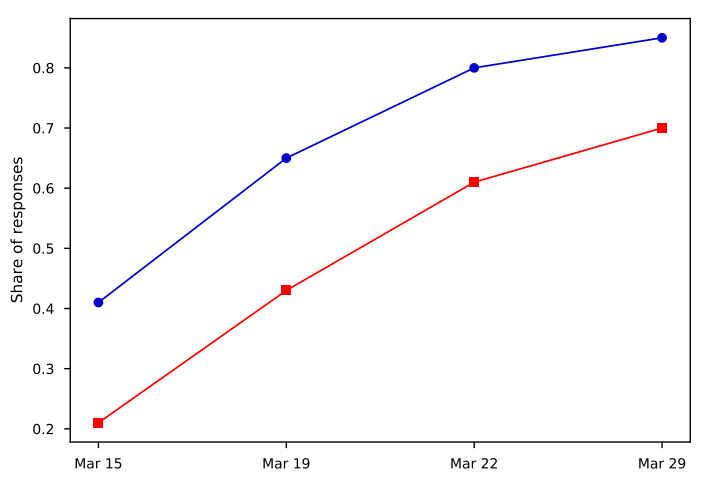

Panel B: Behavior Change from Coronavirus

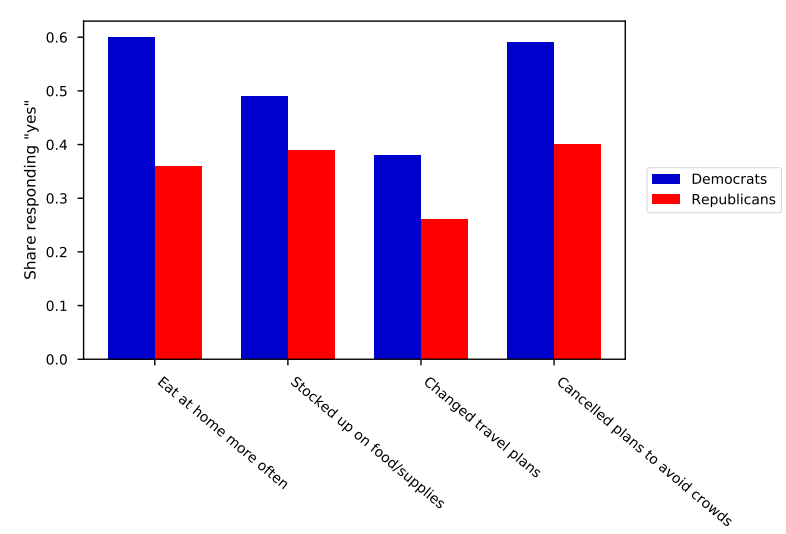

Panel D: Share Avoiding Small Gatherings

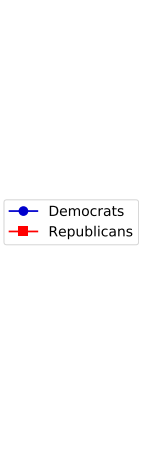

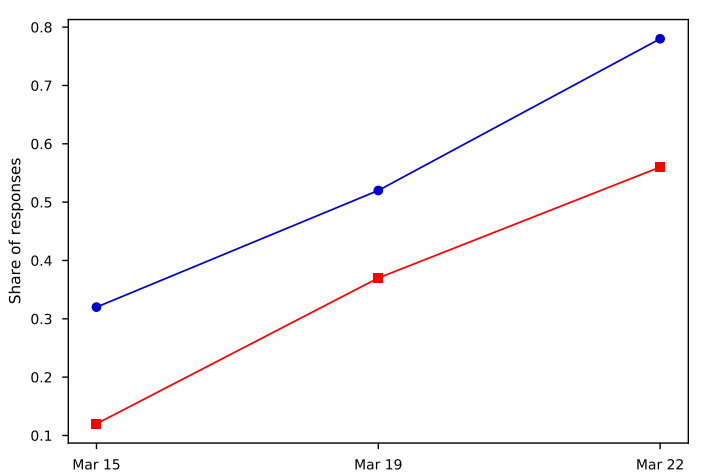

Note: Figure shows responses to nationally representative polls by political affiliation. Panel A shows the share of people concerned about coronavirus spreading to the United States (Piacenza 2020). Panel B shows self-reported behavior change as of March 13-14 (Marist 2020). Panel C shows the share of people avoiding public places, such as stores and restaurants (Saad 2020). Panel D shows that share of people avoiding small gatherings, such as with friends and family (Saad 2020). 

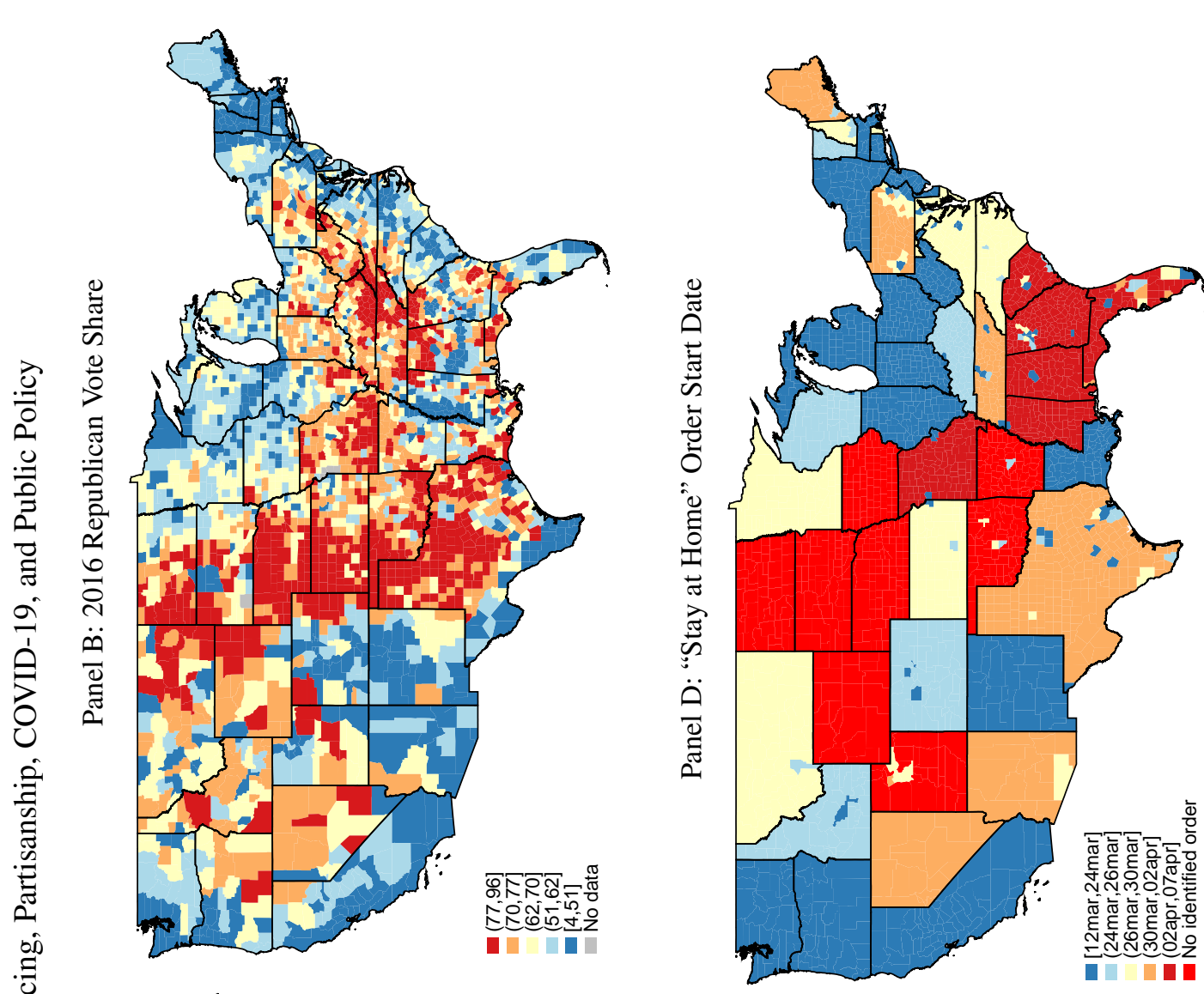

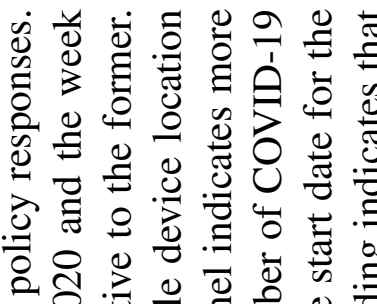

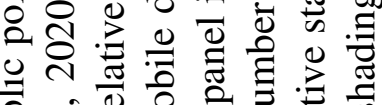

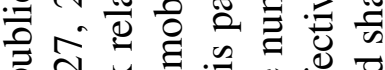

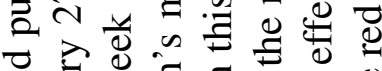

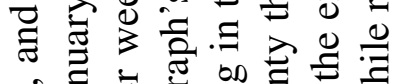

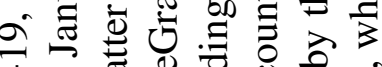
b 00 券

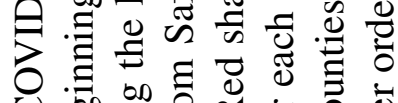
U.

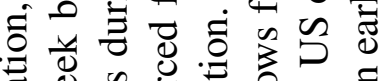

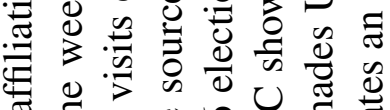

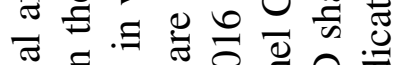

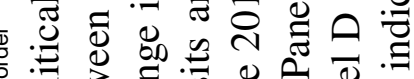

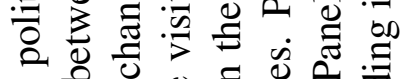
oî

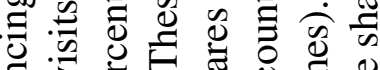
可

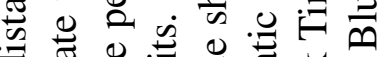

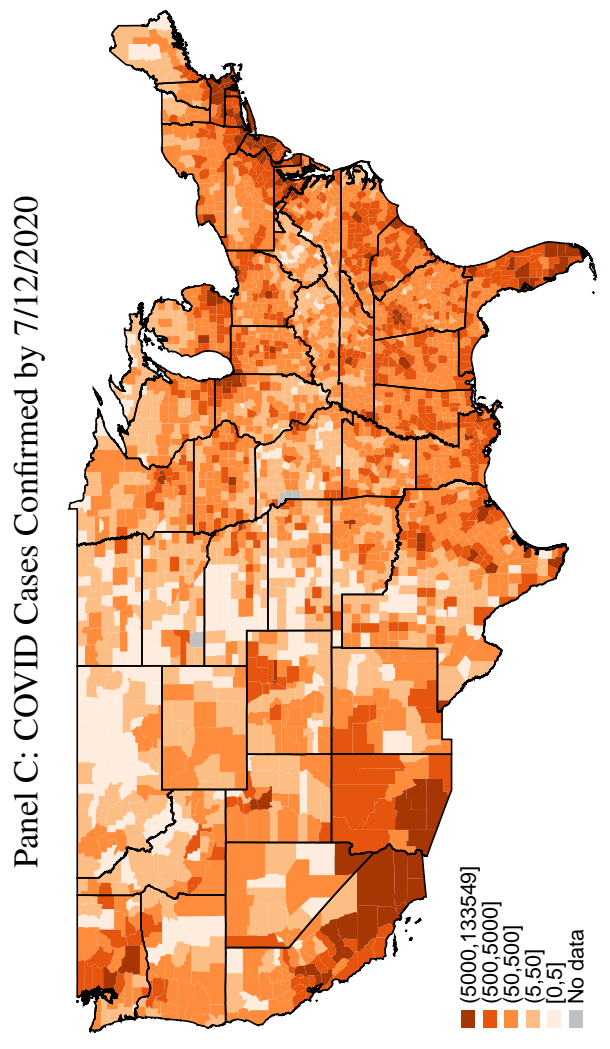

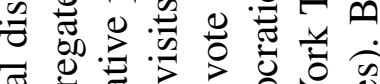

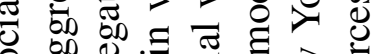
然 $4 . \exists$ o

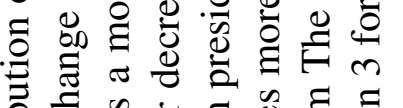
흘

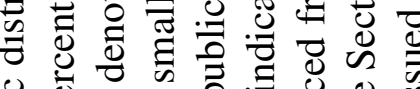

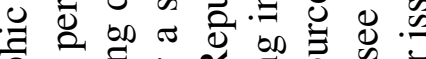

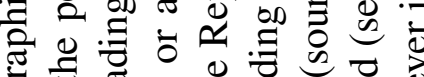
政 品

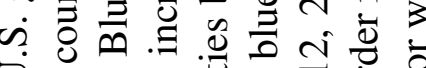
o 点

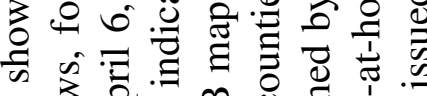
造

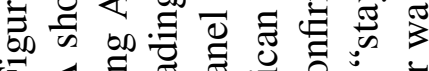

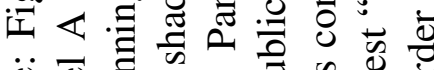

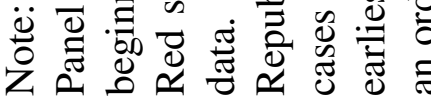


Figure 3: Social Distancing and COVID-19 Incidence

Panel A: POI Visits

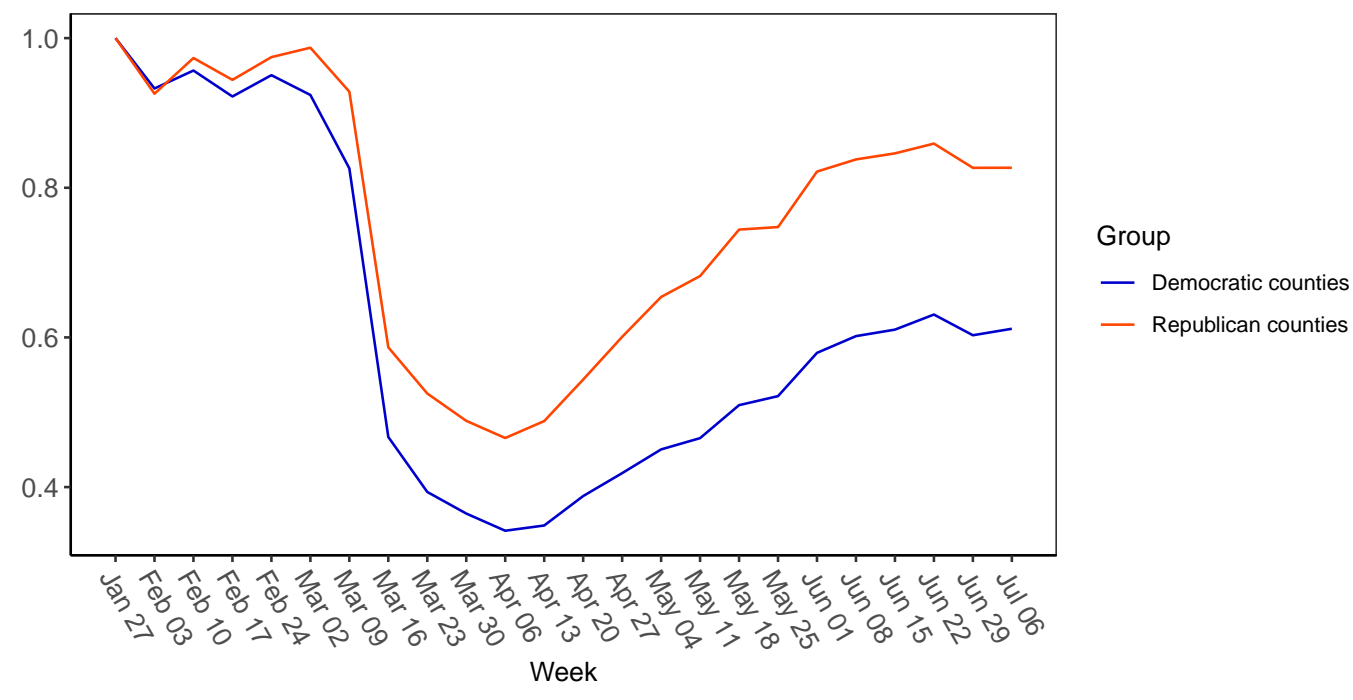

Panel B: Cumulative, Confirmed COVID-19 Cases and Deaths

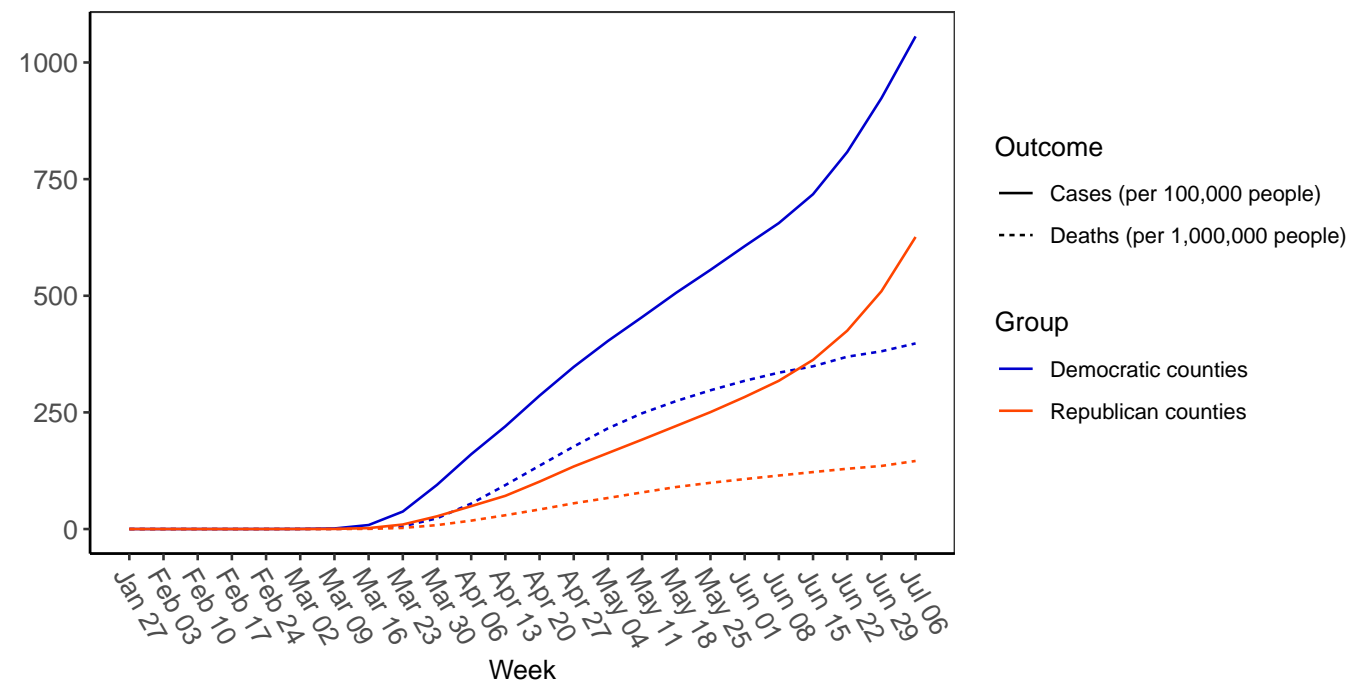

Note: Panel A shows the number of visits (normalized to one) to SafeGraph POIs for each week since January 27, 2020 for Republican counties and Democratic counties separately. Panel B is analogous but plots cumulative, end-of-week values for confirmed COVID-19 cases (per 100,000 people) and confirmed COVID-19 deaths (per 1,000,000 people). Republican counties are defined to be those whose 2016 Republican vote share is greater than the median vote share (66.4 percent) across the counties in our sample. Counties covering New York City, Kansas City, and Alaska are excluded from these counts, as noted in Appendix A.1.1. 
Figure 4: Partisan Differences in Social Distancing

Panel A: Only County \& Time FE

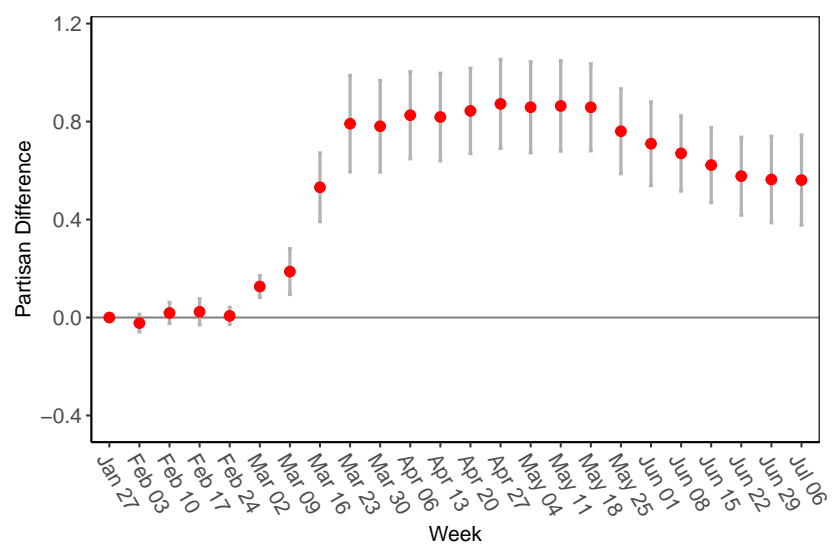

Panel B: Adds State-Time FE

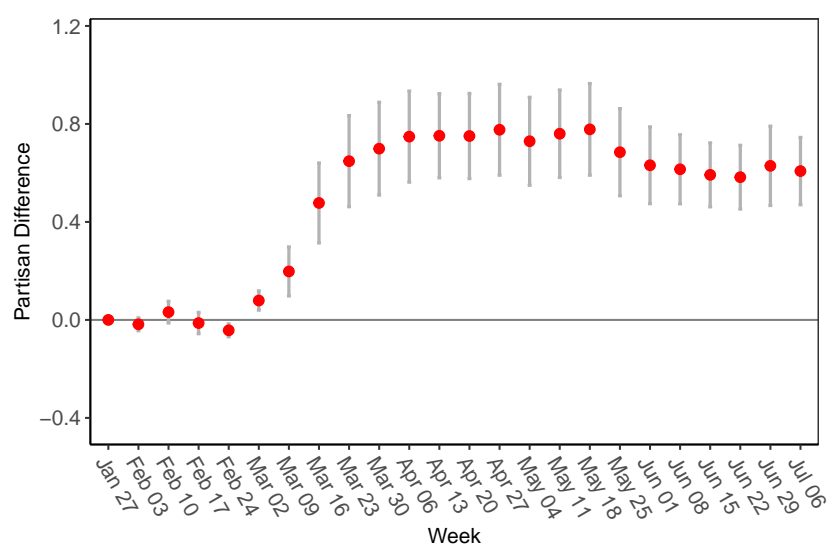

Panel C: Adds Health + Econ + Weather Controls

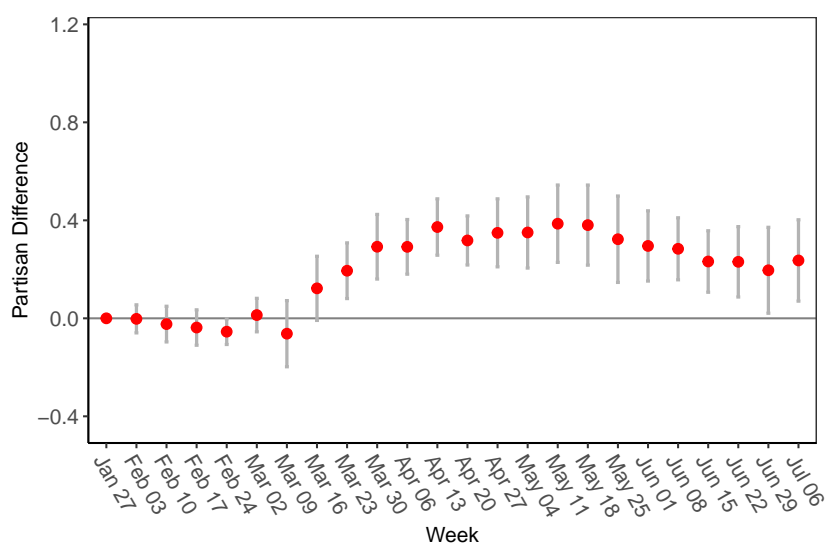

Note: Figure shows the estimated coefficients for county Republican vote share $\rho_{i}$ on the $\log$ number of POI visits in the county. For Panel A, only county and time fixed effects are included as controls. Panel B is the same as Panel A except state-time fixed effects replace the time fixed effects. Panel $\mathrm{C}$ is the same as Panel B except that health, economic, and weather covariates are included (flexibly), as described in the main text. The grey error bars indicate 95 percent confidence intervals constructed using standard errors clustered at the state-level. 
Figure 5: Partisan Differences in Beliefs and Actions

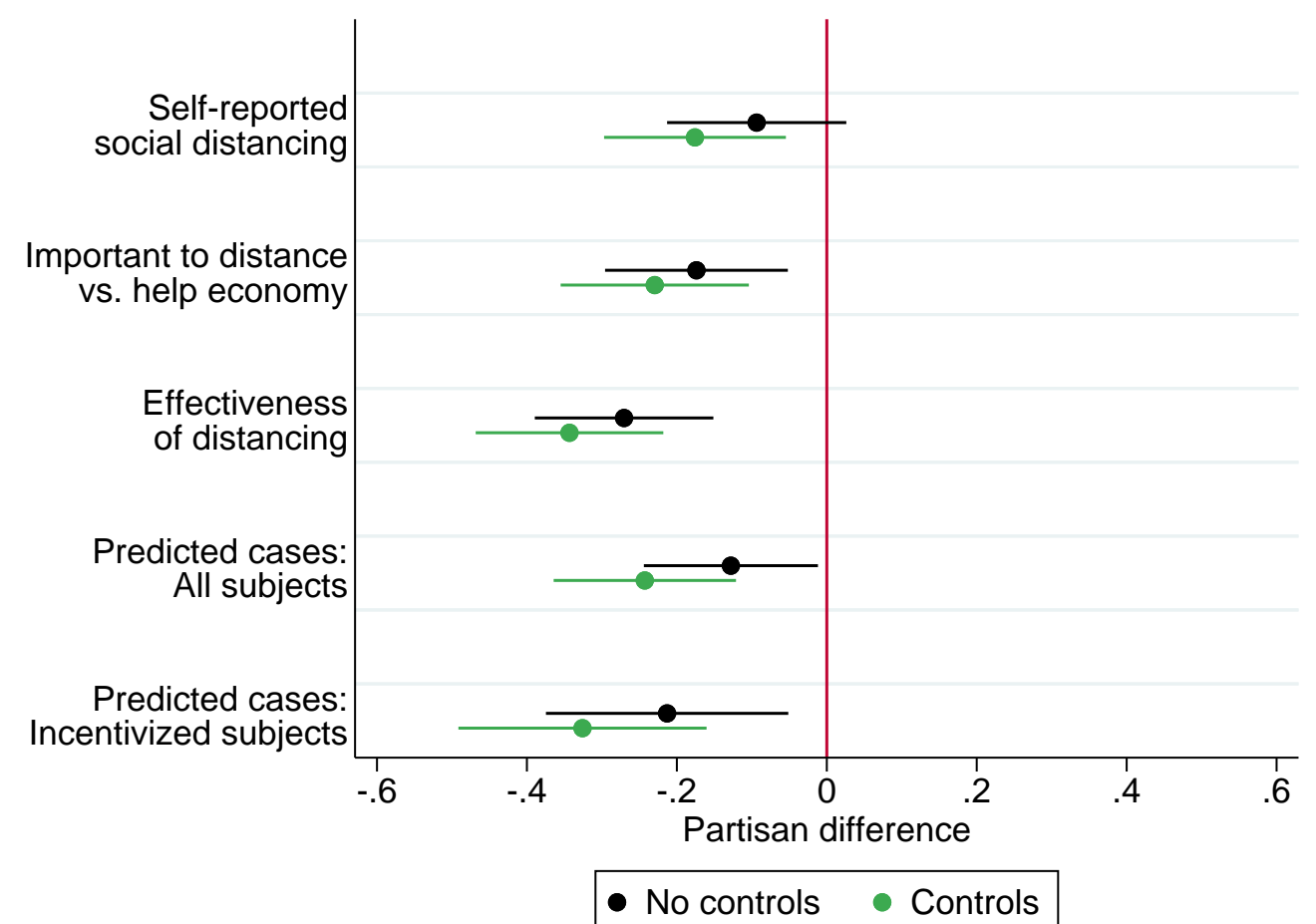

Note: Figure shows coefficient plots from regressing normalized measures of beliefs and actions on our seven-point measure of partisan affiliation which ranges between 0 (Strongly Democratic) and 1 (Strongly Republican). Negative estimates indicate less concern about COVID-19 or social distancing. Demographic controls are age, race, income, education, number of children, $\log$ population at the ZIP code level, county-level deaths and cases, and state fixed effects. 2 percent of observations are set to the mean due to an invalid ZIP code. Self-reported social distancing is the percent reduction in contact with others over one month; effectiveness of distancing is the estimated likelihood of catching COVID-19 in one month without social distancing; importance of distancing vs. economy is subjects' perception of whether it is more important to go out and stimulate the economy versus staying in and preventing the spread of COVID-19; predicted cases are predictions about the number of new COVID-19 cases in the US in April; incentivized subjects restrict to the subsample whose answers are incentivized. Observations are weighted to mimic a representative sample as described in the text. Error bars represent 95 percent confidence intervals. 


\section{A Appendix}

\section{A.1 Data Details}

See replication code for exact details on implementation.

\section{A.1.1 County-Level Data Build}

To construct the county-level dataset used in the analysis, we proceed as follows:

1. We begin by matching SafeGraph POIs to the counties in which they are located. We use latitude and longitude from SafeGraph's July 2020 Core POI dataset, along with the 2010 TIGER county shapefile. ${ }^{21}$ We successfully assign 99.9 percent of the POIs to a county.

2. We then merge the POI-county mapping from (1) onto SafeGraph's Patterns data using the safegraph-place-id variable. We sum visits by county for a given day, aggregating across POIs.

3. We then merge alternative county-day measures of social distancing onto the output from (2). These measures are constructed as follows from the Daily Social Distancing SafeGraph data with observations at the census block group-day level for January 27 through July 12. We exclude Alaska. We restrict our sample to census block groups with active devices throughout the entire time period. We also drop one census block group with anomalous behavior as notified by SafeGraph (FIPS: 190570010001). We aggregate this series to the county level. Countable variables (e.g., 'device count') are summed, while we take a 'device count' weighted average of other variables (e.g., 'median home dwell time').

4. We then merge gridMET weather data onto the output from (3). Precipitation and temperature means for a given county day are taken as a mean across grid cell points that lie within a county boundary. Weather data was not available for Hawaii, so this particular state is dropped in regressions including weather controls.

5. We then merge The New York Times COVID-19 tracking data onto our output from (4). We assume zero cases and deaths for the observations not observed in The New York Times data. We drop the five counties associated with New York City and the four counties which overlap with Kansas City (MO), because The New York Times lists these as geographic exceptions where it either does not assign cases to these counties or excludes cases occurring within the city.

\footnotetext{
${ }^{21}$ Downloaded from https://www.census.gov/geo/maps-data/data/cbf/cbf_counties.html on July 24, 2018.
} 
6. We then merge a dataset of county-level shelter-in-place order start dates onto the output from (5) and construct an indicator for whether a county had been subject to a shelter-inplace order by a given date. This dataset of shelter-in-place orders is the same as in Allcott et al. (2020), where its construction is described in detail. It is ultimately sourced from Keystone Strategy, a crowdsourcing effort from Stanford University and the University of Virginia, Hikma Health, and The New York Times.

7. We then aggregate the output from (6) to the county-week level using sums, averages, or start- or end-of-week observations as appropriate.

8. We then merge onto the output from (7) a dataset of county-level demographic information constructed as follows. We use the Open Census data from SafeGraph, aggregating up the data given at the census block group level to the county level. We combine this with data on county 2016 Presidential votes shares (MIT Election Data and Science Lab 2018). We define the Republican vote share to be the share of votes received by the Republican candidate over the sum of votes across all candidates. We exclude counties without valid vote data, which drops Alaska and two additional counties (FIPS: 15005, 51515).

\section{A.1.2 Precinct-Level Data Build}

1. We begin by matching SafeGraph POIs to the precincts in which they are located. We start with the POIs successfully matched in the POI-county mapping from (1) in Section A.1.1 We use POI latitude and longitude along with 2016 precinct-level shapefiles (Voting and Election Science Team 2018). Of these POIs, we successfully match 99.6 percent to a unique precinct in the states covered by these precinct shapefiles and drop the 0.001 percent matched to two precincts. The precinct shapefiles cover the following 42 states: AK, AR, AZ, CA, CO, DC, DE, FL, GA, HI, IA, ID, IL, KS, KY, LA, MA, MD, ME, MI, MN, MO, MT, NC, ND, NE, NH, NM, NV, OK, OR, RI, SC, SD, TN, TX, UT, VA, VT, WA, WI, and WY.

2. We merge the output from (1) with the Patterns dataset from SafeGraph using the safegraphplace-id variable. We sum visits by precinct in a given week, aggregating across POIs.

3. We then merge alternative precinct-day measures of social distancing onto the output from (2). We start with the cleaned census block group level measures produced from (3) in Section A.1.1. We then aggregate our (countable) alternative social distancing variables to the precinct level as follows. We do this by first constructing the geographic intersections formed by our precinct shapefiles and 2019 Tiger census block group shapefiles. ${ }^{22}$ Let $a_{p}$,

\footnotetext{
${ }^{22}$ Downloaded from ftp://ftp2.census.gov/geo/tiger/TIGER2019/BG/ on April 1, 2020.
} 
$a_{b}$, and $a_{b p}$ denote the area of precinct $p$, census block group $b$, and of their intersection respectively. For a given count variable $x_{b}$ given at the block group level, we construct a precinct-level estimate as: $\hat{x}_{p}:=\sum_{b} \frac{a_{b p}}{a_{b}} x_{b}{ }^{23}$ We then form ratios (e.g., 'share leaving home') using these summed precinct-level estimates as needed.

4. We then merge gridMET weather data onto the output from (3). Weather data by precinct day was constructed as a land area weighted average of weather data for overlapping census block groups. Census block group centroids were first associated with the nearest grid cell centroid in gridMET. Weather data was not available for Hawaii, so this particular state is dropped in regressions including weather controls.

5. We then merge The New York Times COVID-19 county-level tracking data onto our output from (4). Precincts were first associated with the county corresponding to the census block group of their largest intersection. We drop the five counties associated with New York City and the four counties which overlap with Kansas City (MO), because The New York Times lists these as geographic exceptions where it either does not assign cases to these counties or excludes cases occurring within the city.

6. We then merge the dataset of county-level shelter-in-place order start dates from (6) in Section A.1.1 onto the output from (5). We construct an indicator for whether a county had been subject to a shelter-in-place order by a given date.

7. We then aggregate the output from (6) to the precinct-week level using sums, averages, or start- or end-of-week observations as appropriate.

8. We then merge onto the output from (7) a dataset of precinct-level demographic information constructed as follows. We start with the Open Census census block group level demographics from (8) in Section A.1.1. We then aggregate countable variables to the precinct-level using the intersection share procedure described in (3). We then form ratios (e.g., "population density' or 'share hispanic') using these summed precinct-level estimates. We then merge precinct-level 2016 Presidential votes shares (Voting and Election Science Team 2018) onto the resulting output, constructed as in A.1.1 step (8). We drop Alaska in order to be consistent with our county construction.

\section{A.2 Survey Details}

\section{A.2.1 Data}

We clean the survey data from Qualtrics as follows:

\footnotetext{
${ }^{23}$ This estimate is exactly correct if a given demographic $x_{b}$ is evenly distributed across a census block group's area.
} 
1. We match participant IDs from Qualtrics with a list of emailed IDs from CloudResearch and drop observations that do not match to remove test subjects. There is one exception, where the ID on Qualtrics did not correctly generate. We find exactly one remaining participant with the same demographics in the CloudResearch, so we keep this participant.

2. We change one miscoded age from .23 to 23 and one miscoded ZIP code from ,43011 to 43011.

3. We merge ZIP code data with 2010 US Census data and match ZIP codes to states to get population density.

4. We match ZIP codes to counties and use the week of March 29-April 4 and get county-level COVID cases and deaths via The New York Times. All ZIP codes in New York City are matched to the city-level cases and deaths since county-level data is unavailable from The New York Times. For analyses, we control for $\log ($ county cases +1$)$ and $\log ($ county deaths $+1)$.

5. We weight observations across age category, gender, race/ethnicity, and party affiliation using the Stata ebalance command. Weights are prespecified in the pre-analysis plan.

6. News sources are numbered in the data in the following order: (1) Network news; (2) Breitbart; (3) CNN; (4) Facebook; (5) Fox News; (6) MSNBC; (7) New York Times; (8) Wall Street Journal; (9) Twitter; (10) Wikipedia; (11) CDC; (12) WHO. News sources (1)-(3) and (5)-(8) are ranked by partisanship as specified in our pre-analysis plan. For our news consumption specification, we use the question on consumption of news about the coronavirus. Often is coded as 1 , Sometimes as 2/3, Rarely as 1/3, and Never/Not Familiar as 0. Answers are then multiplied by the pre-specified partisanship of each source (-1 for NYT; $-2 / 3$ for MSNBC; $-1 / 3$ for CNN; 0 for Network; 1/3 for WSJ; 2/3 for Fox News; 1 for Breitbart). The weighted average of news partisanship for each participant equals the sum of (Answer * Source Partisanship) divided by the sum of Answer.

We have the following demographic groups prior to weighting:

- Age: $45.7 \%$ 18-39, 33.8\% 40-59, $20.5 \% 60+$

- Gender: 51.9\% Female, 47.75\% Male, 0.35\% Other / Non-binary

- Race: $66.6 \%$ White (Not Hispanic or Latinx), 15.25\% Hispanic or Latinx, $11.2 \%$ Black or African American (Not Hispanic or Latinx), 4.95\% Asian or Pacific Islander, $2.0 \%$ Other.

- Party: 34.65\% Democratic, 31.25\% Republican, 32.8\% Independent, 1.3\% Other 


\section{A.2.2 Survey Questions}

Screening

- What is your gender? [Male; Female; Other / Non-binary]

- What race/ethnicity best describes you? [American Indian or Alaska Native; Asian or Pacific Islander; Black or African American (Not Hispanic or Latinx); Hispanic or Latinx; White (Not Hispanic or Latinx); Other]

- Do you consider yourself a Republican, a Democrat, or an Independent? [Democrat (Strongly Democratic); Democrat (Weakly Democratic); Independent (Lean toward the Democratic Party); Independent (Do not lean towards either party); Independent (Lean toward the Republican Party); Republican (Weakly Republican); Republican (Strongly Republican); Other / prefer not to say]

- What is your age?

- Do you currently live in the United States? [Yes; No]

\section{Consent}

[Page seen if age $>18$, United States $=$ Yes, and not screened out due to demographic quotas.]

Congratulations! You are eligible to participate. Please read the consent form below:

DESCRIPTION: You are invited to participate in an online research study on your views about the news and predictions of what will happen in the future. This is a research project being conducted by researchers at Harvard University and New York University.

TIME INVOLVEMENT: Your participation will take approximately 20 minutes, and the entire study will take place online.

RISKS AND BENEFITS: We will ensure that your individual responses are strictly confidential, and research results will only be presented in the aggregate. Your responses will not be shared with government officials or any 3rd party. We hope that the knowledge gained from this study will benefit society in general. We cannot and do not guarantee or promise that you will receive any direct benefits from this study.

PAYMENTS: If you are eligible for the study, and once you complete the study, you will receive a participation fee. You may also earn a bonus payment of up to $\$ 100$ via an Amazon gift card. All payments will be through your research provider.

PARTICIPANT'S RIGHTS: If you have read this form and have decided to participate in this project, please understand your participation is voluntary and you have the right to withdraw your consent or discontinue participation at any time without penalty or loss of benefits 
to which you are otherwise entitled. The alternative is not to participate. You have the right to refuse to answer particular questions. The results of this research study may be presented at scientific or professional meetings or published in scientific journals.

\section{CONTACT INFORMATION:}

Questions: If you have any questions, concerns or complaints about this research, its procedures, risks and benefits, contact the researchers at rb4337@nyu.edu.

Independent Contact: If you are not satisfied with how this study is being conducted, or if you have any concerns, complaints, or general questions about the research or your rights as a participant, please contact the Harvard University Area Institutional Review Board (IRB) to speak to someone independent of the research team at cuhs@ harvard.edu, (617)-496-2847. You can also write to the Committee on the Use of Human Subjects, Harvard University, 44-R Brattle Street, Suite 200, Cambridge, MA 02138.

\section{Please retain a copy of this form for your records.}

If you wish to participate in this study, please click "I consent" to proceed. This serves as an electronic signature indicating your consent to participate in the study.

[I consent; I do not consent]

[Only consenting subjects proceed]

\section{Demographics}

- How many children under the age of 18 do you have? $[0 ; 1 ; 2 ; 3 ; 4 ; 5$ or more]

- What is the highest degree or level of schooling that you have completed? [Less than a high school diploma; High school diploma or equivalent (for example: GED); Some college but no degree; Associate's degree; Bachelor's degree; Graduate degree (for example: MA, MBA, JD, $\mathrm{PhD})]$

- What was your total income in 2019? Please include only employment income (wages, salary, bonuses, tips, and any income from your own businesses). [I did not earn income in $2019 ; \$ 1$ to $\$ 9,999 ; \ldots ; \$ 50,000$ to $\$ 59,999 ; \$ 60,000$ to $\$ 74,999 ; \$ 75,000$ to $\$ 99,999$; $\$ 100,000$ to $\$ 124,999 ; \$ 125,000$ to $\$ 149,999 ; \$ 150,000$ or more] [Coded as midpoint of range in thousands of dollars except for top bracket, who is coded at 200. $\log ($ income +1$)$ is used as the control.]

- In what ZIP Code do you currently live? Please enter your 5-digit ZIP Code.

- In general, how would you rate your OVERALL health? [Excellent / Very good / Good / Fair / Poor] 
- Has a doctor ever told you that you had the following conditions? [Yes / No]

- Diabetes or high blood sugar

- Lung disease such as chronic bronchitis or emphysema

- A heart attack, coronary heart disease, angina, congestive heart failure, or other heart problems

- Please answer the following yes/no questions:

- In the past week, have you had to go to a work environment in which you were within six feet of others?

- Have you smoked at least 100 cigarettes in your entire life?

- Have you smoked at least 10 cigarettes in the past week?

\section{Information sources}

- All of the following questions were asked about the following 12 news sources: Network news (ABC, CBS, NBC); Breitbart; CNN; Facebook; Fox News; MSNBC; The New York Times; The Wall Street Journal, Twitter, Wikipedia, The Centers for Disease Control (CDC); The World Health Organization (WHO).

- Last year, how much trust and confidence did you have in each of the following sources when it comes to reporting about politics and current events fully, accurately, and fairly? [A great deal / A fair amount / Not very much / None at all / Not familiar with this outlet]

- Last year, how frequently did you get news and information from each of the following sources about politics and current events through any medium (including reading online, watching on TV, etc.)? [Often / Sometimes / Rarely / Never / Not familiar with this outlet]

- How much trust and confidence do you have in each of the following sources when it comes to reporting about the coronavirus fully, accurately, and fairly? [A great deal / A fair amount / Not very much / None at all / Not familiar with this outlet]

- How frequently are you getting news and information from each of the following sources about the coronavirus through any medium (including reading online, watching on TV, etc.)? [Often / Sometimes / Rarely / Never / Not familiar with this outlet] 
Changes in behavior and effects of social distancing

- Think about the ways you may have changed your daily routine in the past two weeks specifically because of the coronavirus. For example, you may be washing your hands more, avoiding restaurants and other public places, and/or reducing interactions with friends and family.

- By what percent have you reduced your overall contact with other people as a result of the coronavirus outbreak? Please enter a percentage from 0 to 100.

- Think back to two weeks ago.

- As of two weeks ago, by what percent had you reduced your overall contact with other people as a result of the coronavirus outbreak? Please enter a percentage from 0 to 100.

- Imagine that starting today and for the rest of the month, you went back to your normal daily routine from before the coronavirus. What do you think is the probability that you would catch the coronavirus in the next month? Please enter a percentage from 0 to 100. [Subjects who answer 0 for the percent reduction question see "continued with" instead of "went back to.']

- Imagine that starting today and for the next month, you cut off all in-person contact with people outside your household. What do you think is the probability that you would catch the coronavirus in the next month? Please enter a percentage from 0 to 100.

- We'd like to quantify the overall costs (in terms of time, money, and inconvenience) that social distancing imposes on you. Consider a hypothetical situation in a normal month in the future, after the coronavirus outbreak is completely over.

Imagine you had a choice between:

(A) following your normal routine for one month,

OR

(B) cutting off all in-person contact with people outside your household for one month, AND receiving $\$ \mathrm{X}$ cash.

Presumably if you were offered a large amount of cash ( $\$ X$ is large), you'd be willing to cut off all social contact. If you weren't offered any cash ( $\$ X$ is 0$)$, you'd prefer to stick with your normal routine. What value of $\mathrm{X}$ would make you equally happy with these two options? Please answer in dollars. 
Economic trade-offs

- When there was no "stay-at-home" order for your area, what did you think was the best way to help the country in this time of crisis? [7-point scale from "Go out more to help the economy" to "Go out less to avoid spreading the coronavirus"]

\section{Predictions}

[If unincentivized:]

- You will now be asked to make a few predictions.

[If incentivized:]

- You will now be asked to make a few predictions. Think carefully! We'll randomly select 10 participants for an accuracy reward. If you're selected, we'll pay you up to $\$ 100$ depending on how accurate your prediction was. For example:

- If your answer is exactly right, we'll give you $\$ 100$

- If your answer is $1 \%$ off, we'll give you $\$ 99$

- If your answer is $2 \%$ off, we'll give you $\$ 98$

$-\ldots$

- If your answer is $50 \%$ off, we'll give you $\$ 50$

- etc.

All subjects see:

- We want to know how well you think the U.S. will limit the spread of the coronavirus in the next month. There had been 177,226 known cases of coronavirus in the U.S. by March 31 . How many additional known cases will there be in the U.S. in the month of April?

- RealClearPolitics reports polling data on public approval of President Trump's handling of the coronavirus outbreak. What percent of people will say they approve of Trump's handling of the coronavirus outbreak on the latest poll that ends before April 30? 


\section{Appendix Figure A1: POI Visits in 2019}

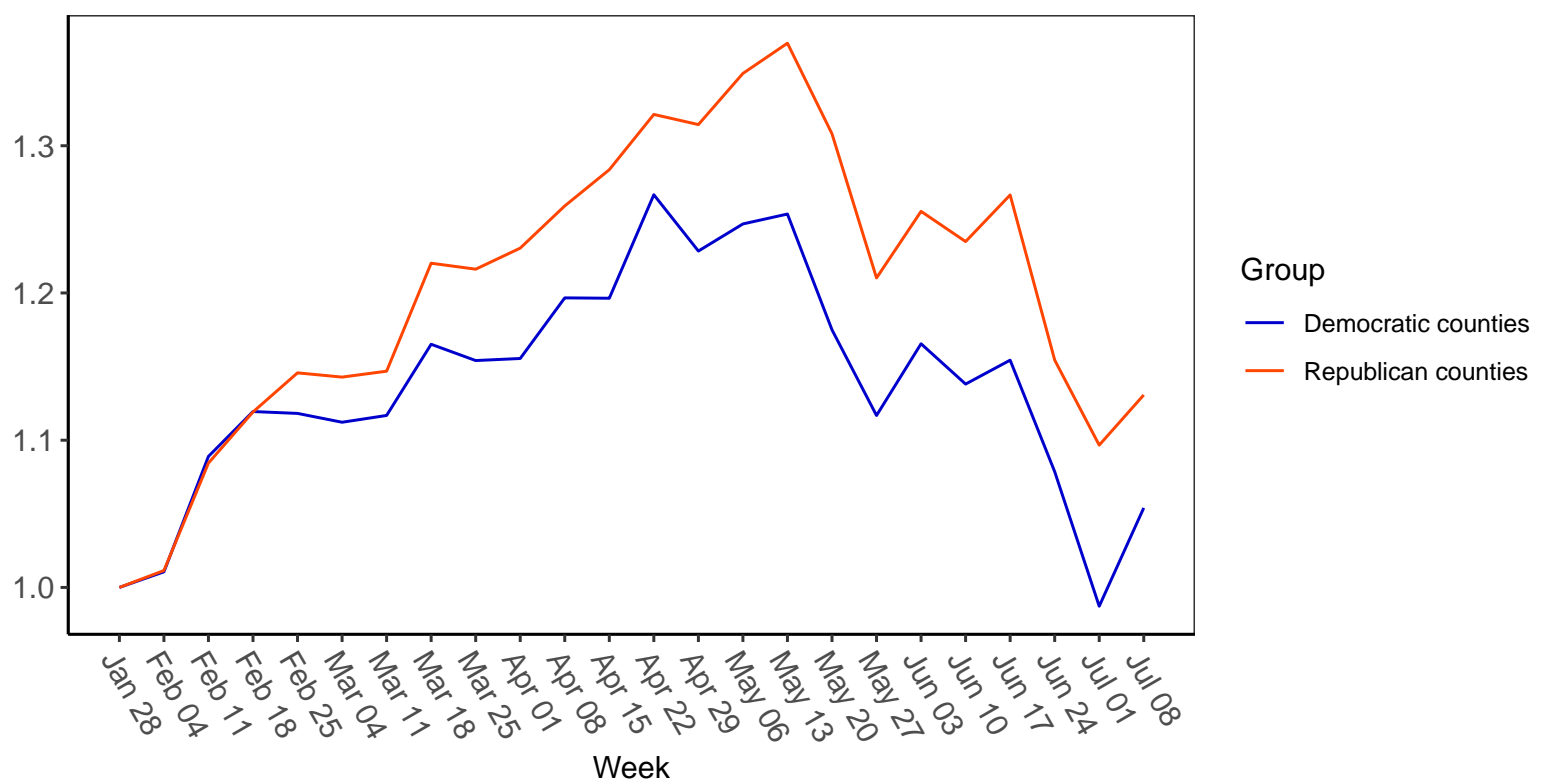

Note: Figure shows the aggregate number of POI visits (normalized to one) for 24 weeks starting on January 28,2019 for Republican counties and Democratic counties. Republican counties are defined to be those whose 2016 Republican vote share is greater than the median vote share (66.4\%) across the counties in our sample. Counties covering New York City, Kansas City, and Alaska are excluded from these counts, as in Figure 3 and as noted in Section A.1.1 


\section{Appendix Figure A2: Partisan Differences in Social Distancing, 2019}

Panel A: Only County \& Time FE

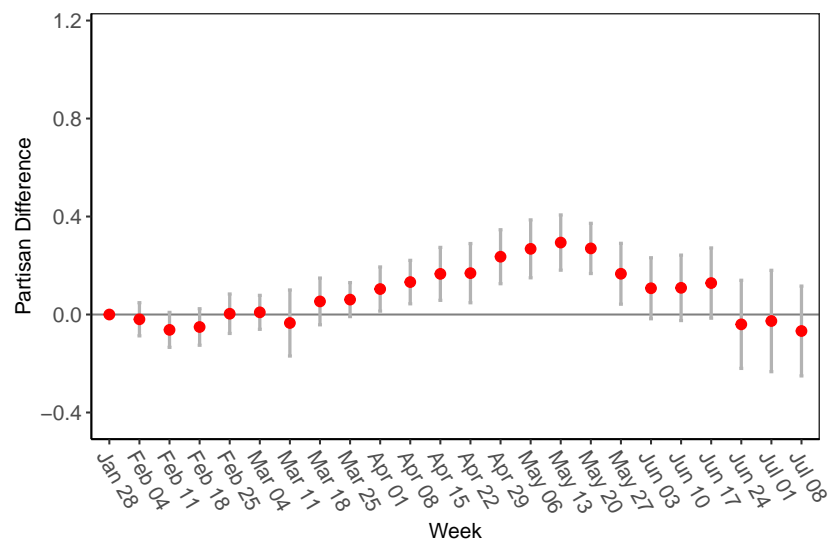

Panel B: Adds State-Time FE

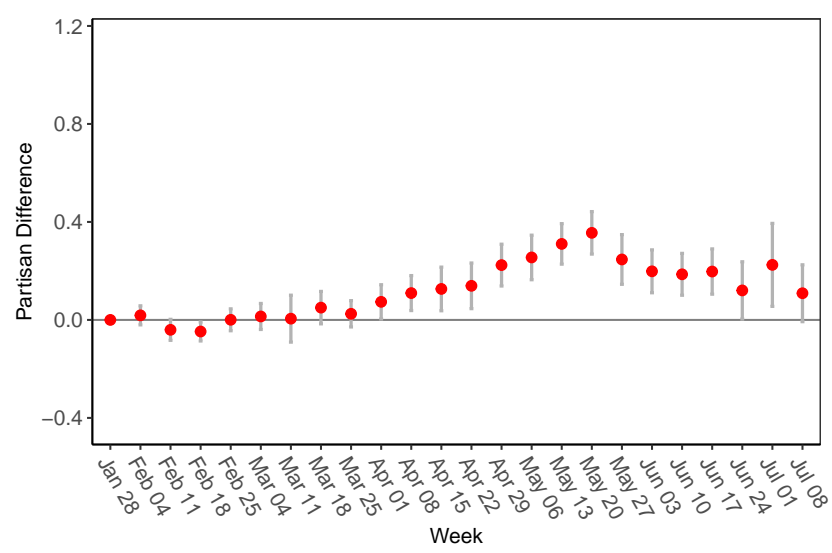

Panel C: Adds Health + Econ + Weather Controls

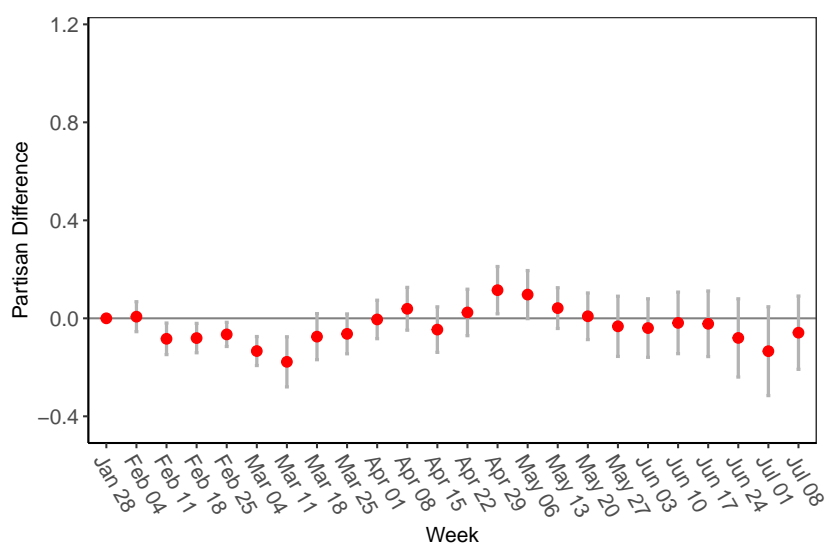

Note: Figure shows the estimated coefficients for county Republican vote share $\rho_{i}$ on the log number of POI visits in the county as in Figure 4 except that 24 weeks of data from January 28, 2019 are used instead of January 27, 2020. For Panel A, only county and time fixed effects are included as controls. Panel B is the same as Panel A except state-time fixed effects replace the time fixed effects. Panel C is the same as Panel B except that health, economic, and weather covariates are included (flexibly), as described in the main text. The grey error bars indicate 95 percent confidence intervals constructed using standard errors clustered at the state-level. 
Appendix Figure A3: Partisan Differences in Social Distancing, Alternative Specifications

\section{Panel A: Dropping Controls}

Drops Health Controls

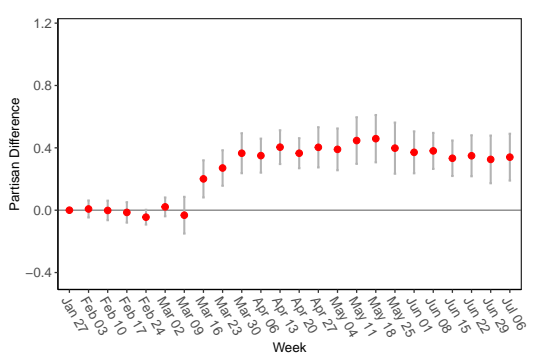

Drops Economic Controls

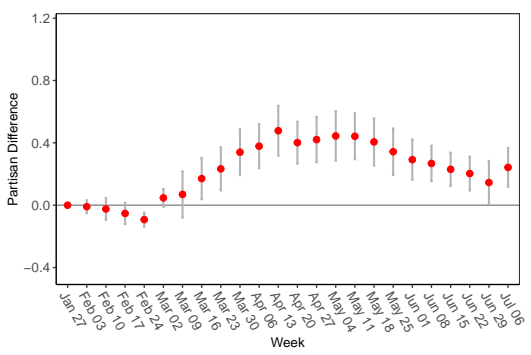

Drops Weather Controls

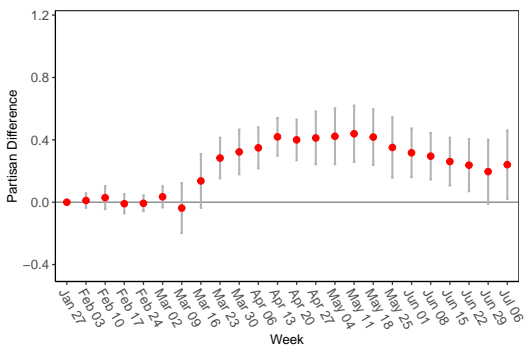

\section{Panel B: Additional Specifications}

Drops State-Time FE

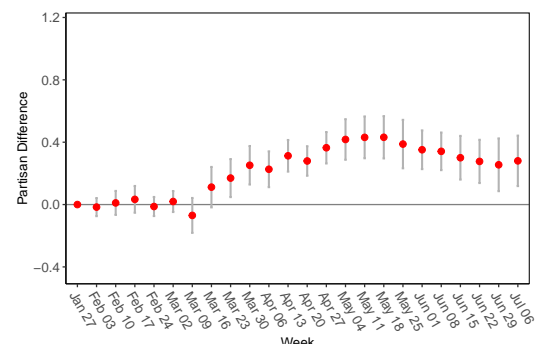

Linear Controls $\times$ Time FE

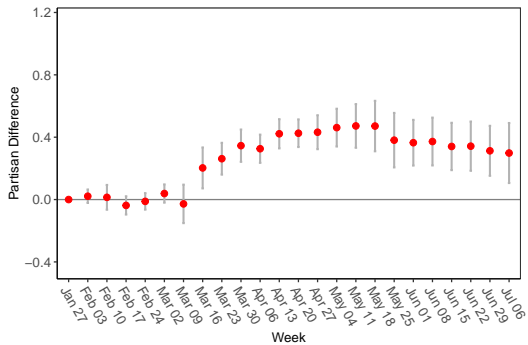

Adds Additional Controls

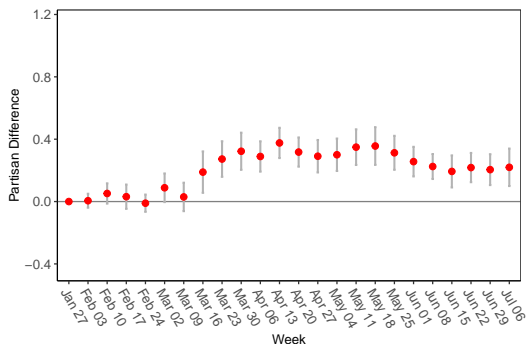

\section{Panel C: Partisanship Indicators}

Above or Below Median

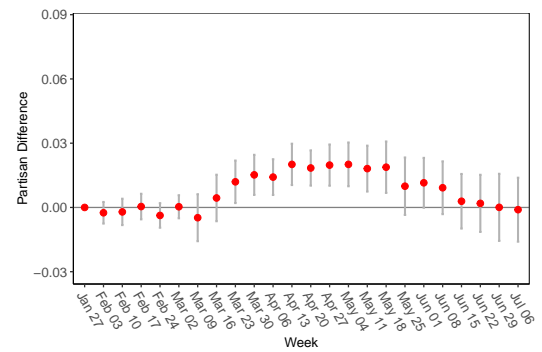

Top or Bottom Quartile

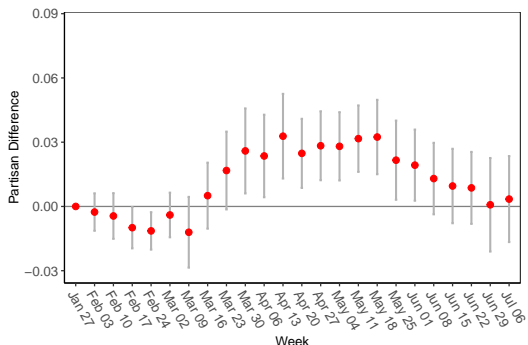

Top or Bottom Decile

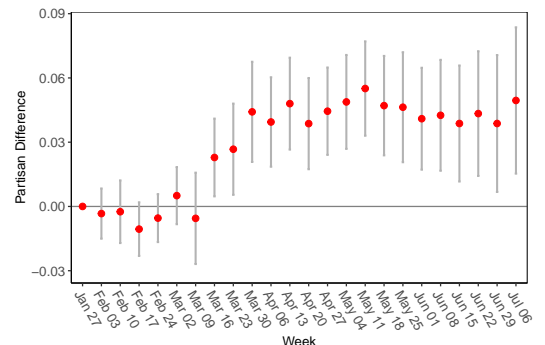

Note: Figure shows the estimated coefficients for county Republican vote share $\rho_{i}$ on the log number of POI visits in the county. The specifications are analogous to our baseline in Panel C of Figure 4 except for the following deviations.

- Panel A: The first plot drops the health controls; the second plot drops the economic controls; and the third plot drops the weather controls.

- Panel B: The first plot drops state-time fixed effects (keeping county fixed effects and time fixed effects); the second plot interacts time fixed effects with linear versions of all controls, rather than with decile indicators; the third plot adds the following controls, each with time-varying decile indicators: share under age 18; shares with degrees in science+engineering, business, or arts+humanities; share of households with at least one vehicle; share of homes rented; share speaking only English at home; share with health insurance; shares commuting by auto, taxi, cycle, walking, or without commute; share enrolled in grad+professional school; share citizens; share married; shared of households with an age 60+ occupant; shares of households of size 1, 2, or 3-5; share of households which are a family. This third plot also adds as a control the log of POI visits (plus one) in the same week and county but during the previous year, included as a linear control interacted with time fixed effects.

- Panel C: The first plot defines partisanship $\rho_{i}$ to be 1 if the Republican vote share is greater than the median and -1 otherwise; the second plot defines partisanship $\rho_{i}$ to be 1 if the Republican vote share is in the top quartile, -1 if in the bottom quartile, and 0 otherwise; and the third plot defines partisanship $\rho_{i}$ to be 1 if the Republican vote share is in the top decile, -1 if in the bottom decile, and 0 otherwise. 
Appendix Figure A3: Partisan Differences in Social Distancing, Alternative Specifications cont.

\section{Panel D: Sample Restrictions by Population and State}

Population above 3,000

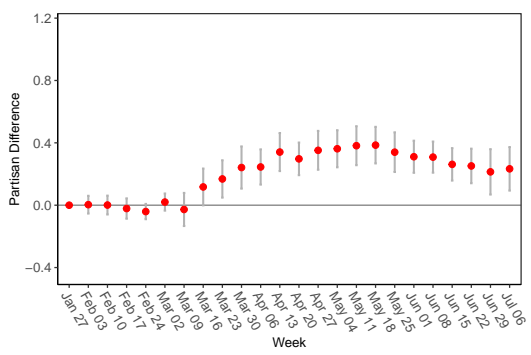

Population below 500,000

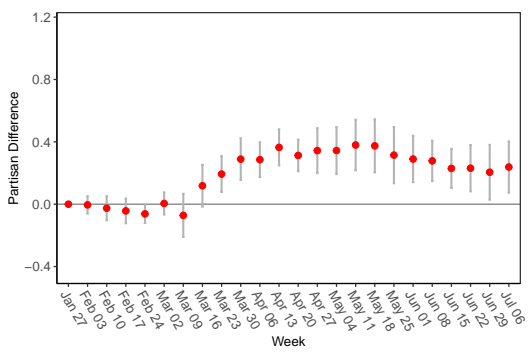

Drops CA, WA, and NY

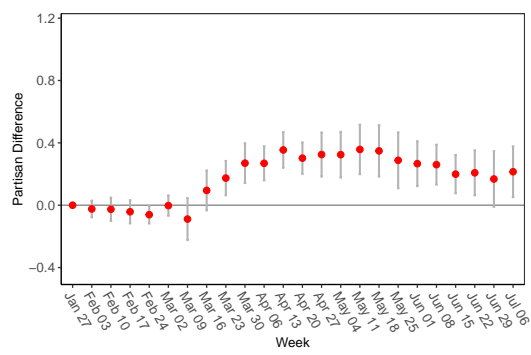

Panel E: Sample Restrictions by Republican Vote Share

Drops Bottom and Top Deciles

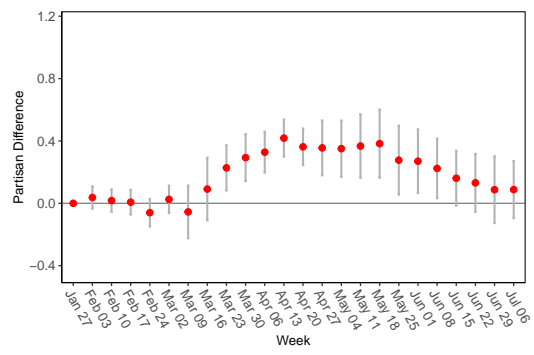

Above Median Vote Share

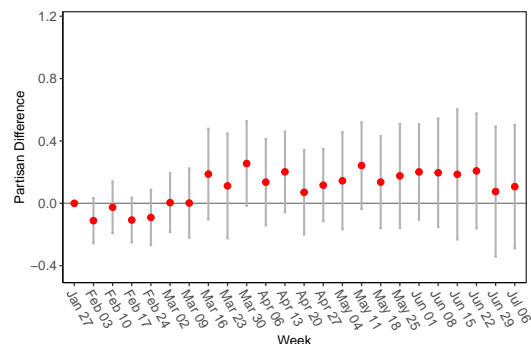

Below Median Vote Share

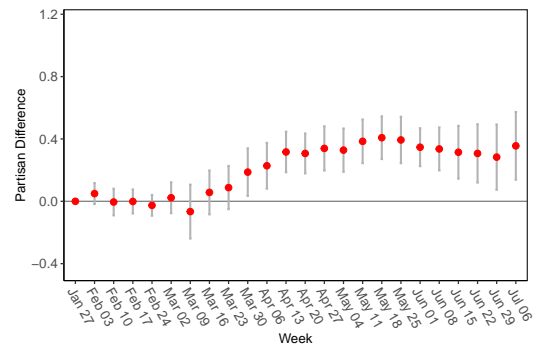

Panel F: Weighting, County Clustering, and Alternative Start Date

Weights by Population

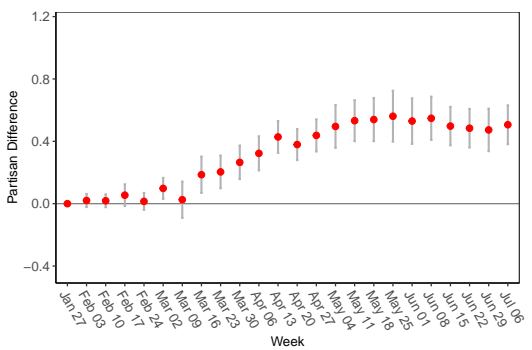

Clusters SEs by County

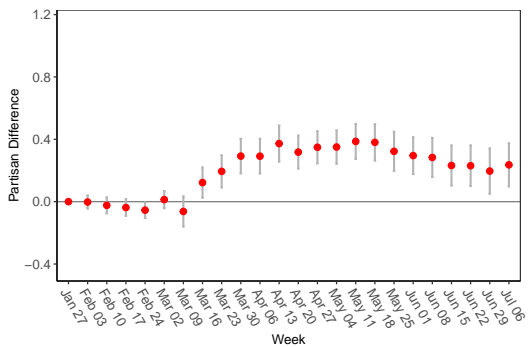

Drops Week of January 27

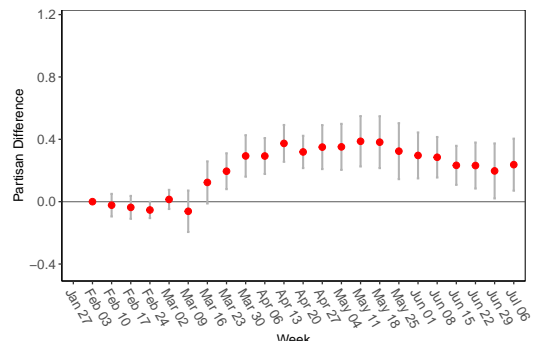

Note: Figure shows the estimated coefficients for county Republican vote share $\rho_{i}$ on the log number of POI visits in the county. The specifications are analogous to our baseline in Panel $\mathrm{C}$ of Figure 4 except with the following deviations.

- Panel D: The first plot only keeps counties with a population above 3,000 (dropping 5.1 percent of counties in our sample); the second plot only keeps counties with a population below 500,000 (dropping 4.1 percent of counties in our sample); the third plot drops California, Washington, and New York.

- Panel E: The first plot drops counties for which the Republican vote share was in the bottom or top decile; the second plot keeps counties for which the Republican vote share is greater than the median; and the third plot keeps counties for which the Republican vote share is less than or equal to the median.

- Panel F: The first plot weights observations by the county's population. The second plot clusters standard errors at the county level. The third plot drops the week of January 27 and normalizes the estimates relative to the week of February 3. 


\section{Appendix Figure A4: Partisan Differences in Social Distancing, Precinct}

(A) Precinct + Time FE

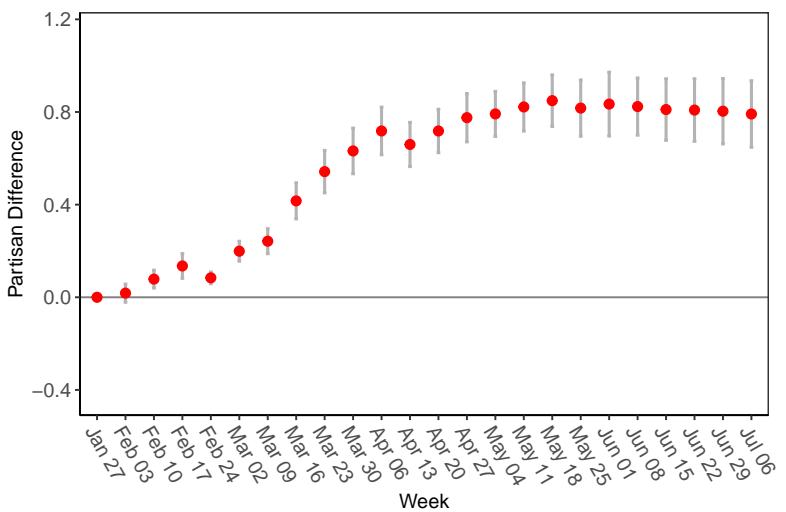

(C) Adds Health + Econ + Weather Controls

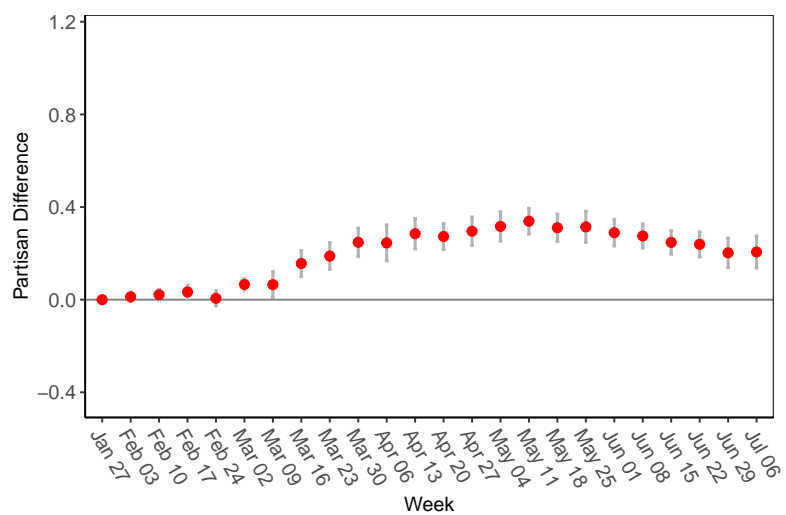

(B) Adds State-Time FE

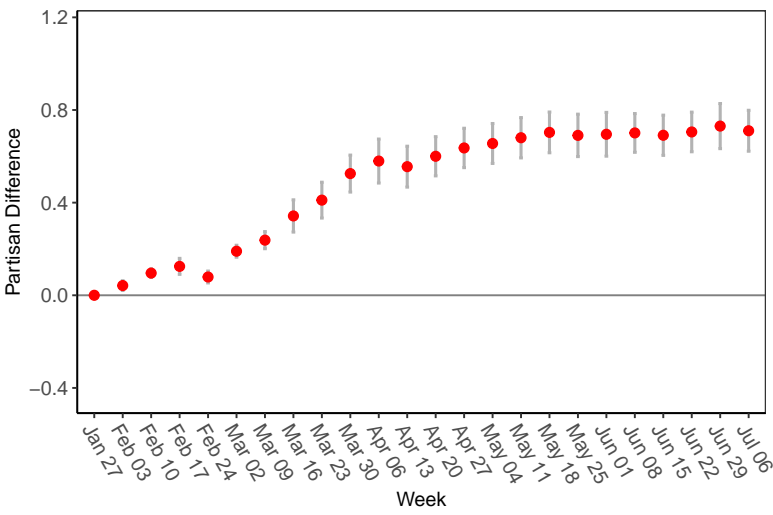

(D) Adds County-Time FE

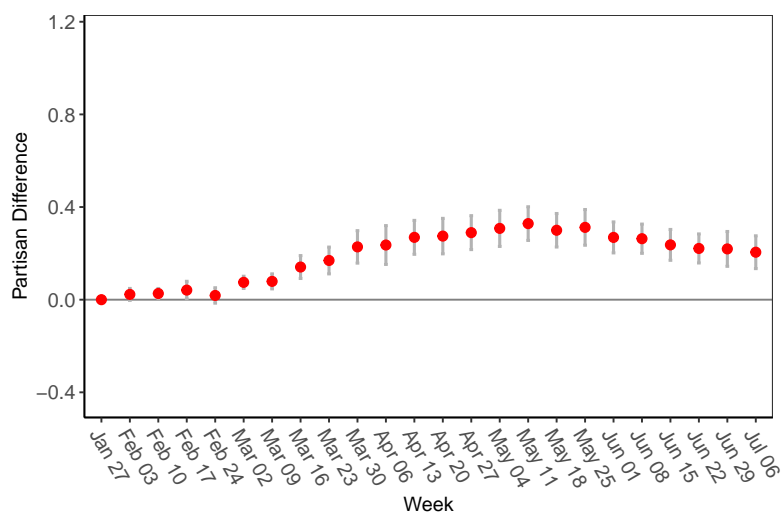

Note: Figure shows the estimated coefficients for precinct partisanship $\rho_{i}$ on the $\log$ number of POI visits in the precinct using the specification outlined in the main text. For Panel A, only precinct and time fixed effects are included as controls. Panel B is the same as Panel A except state-time fixed effects replace the time fixed effects. Panel C is the same as Panel B except that health, economic, and weather covariates are included (flexibly), as described in the main text. Panel D is the same as Panel C except that county-time fixed effects replace the state-time fixed effects. The county-level COVID-19 controls are also subsumed in this specification. The grey error bars indicate 95 percent confidence intervals constructed using standard errors clustered at the state-level. See footnote 14 for limitations regarding this precinct-level analysis. 


\section{Appendix Figure A5: Partisan Differences in Social Distancing, Precinct 2019}

(A) Precinct + Time FE

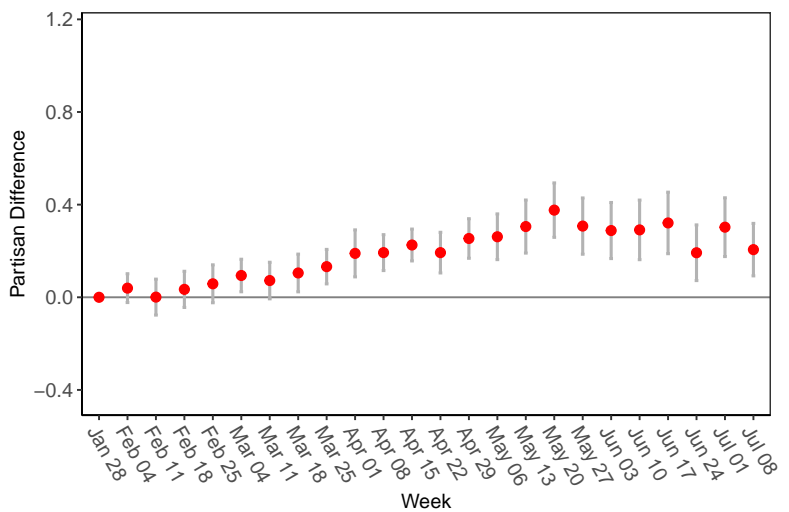

(C) Adds Health + Econ + Weather Controls

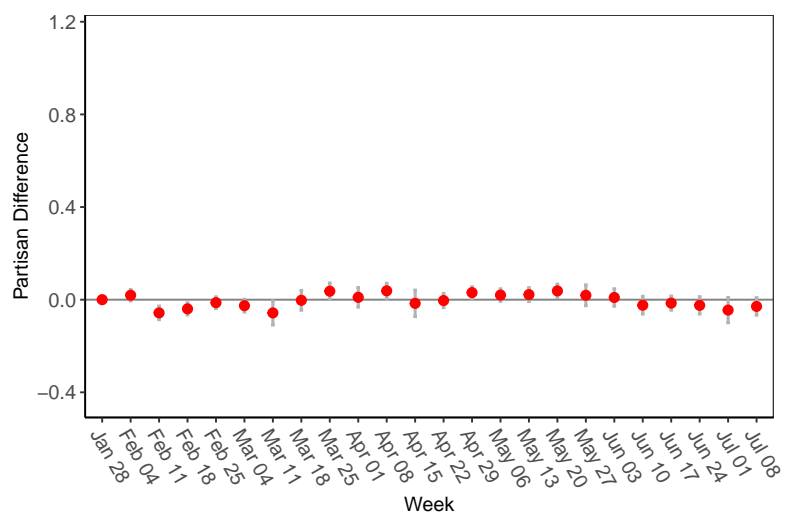

(B) Adds State-Time FE

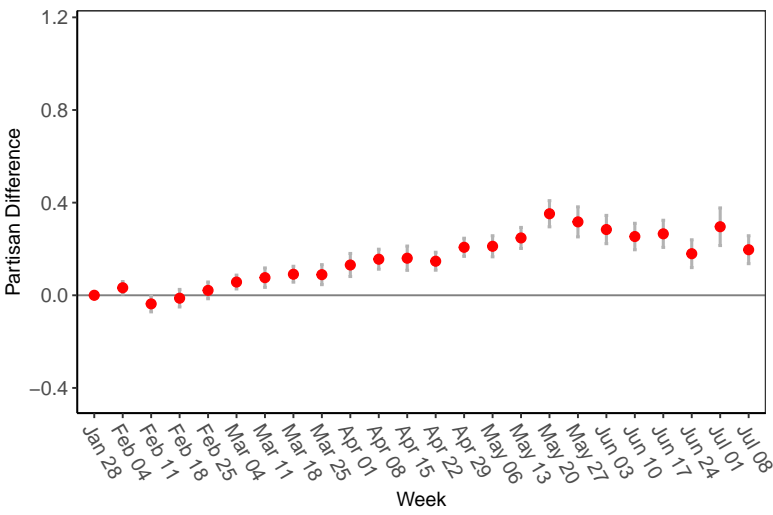

(D) Adds County-Time FE

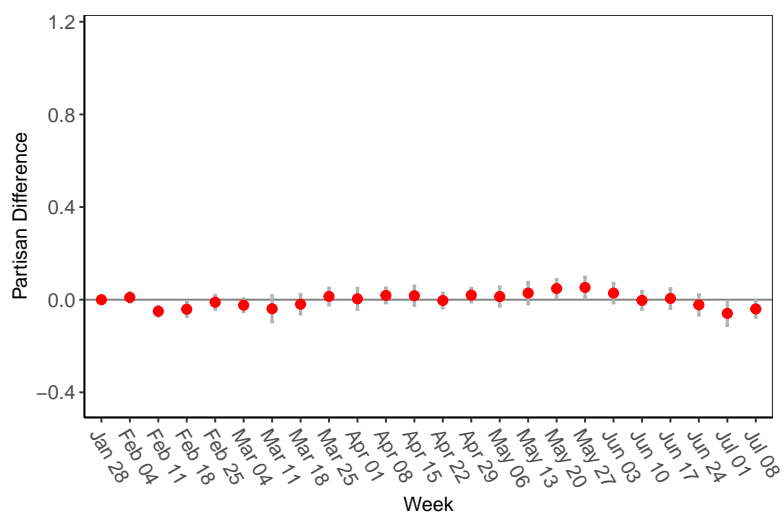

Note: Figure shows the estimated coefficients for precinct partisanship $\rho_{i}$ on the log number of POI visits in the precinct. The figure mirrors Appendix Figure A4 except that 24 weeks of data from January 28, 2019 are used instead of January 27, 2020. For Panel A, only precinct and time fixed effects are included as controls. Panel B is the same as Panel A except state-time fixed effects replace the time fixed effects. Panel C is the same as Panel B except that health, economic, and weather covariates are included (flexibly), as described in the main text. Panel D is the same as Panel $\mathrm{C}$ except that county-time fixed effects replace the state-time fixed effects, the county-level COVID-19 controls are also dropped in this specification. The grey error bars indicate 95 percent confidence intervals constructed using standard errors clustered at the state-level. See footnote 14 for limitations regarding this precinct-level analysis. 


\section{Appendix Figure A6: Partisan Differences in Social Distancing by 2-Digit NAICS Code Industry}

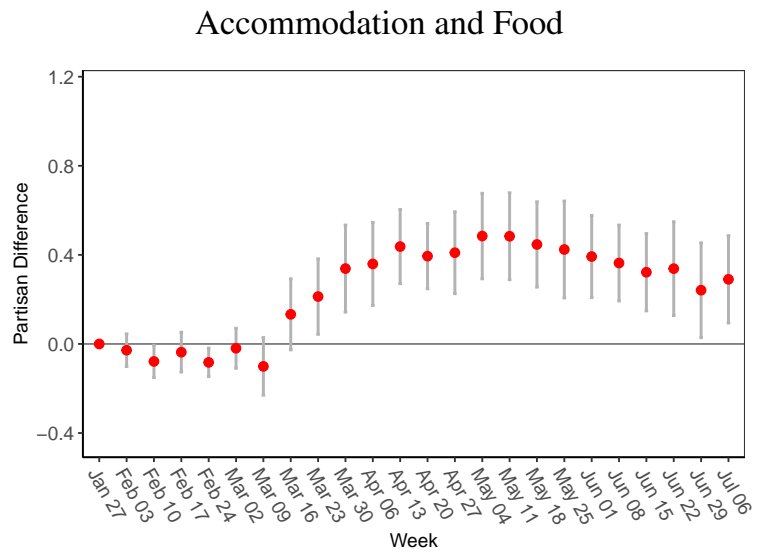

Retail

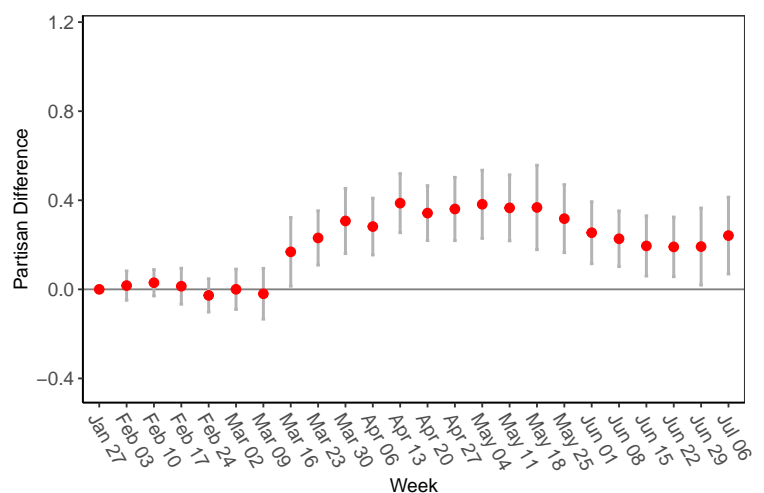

Other

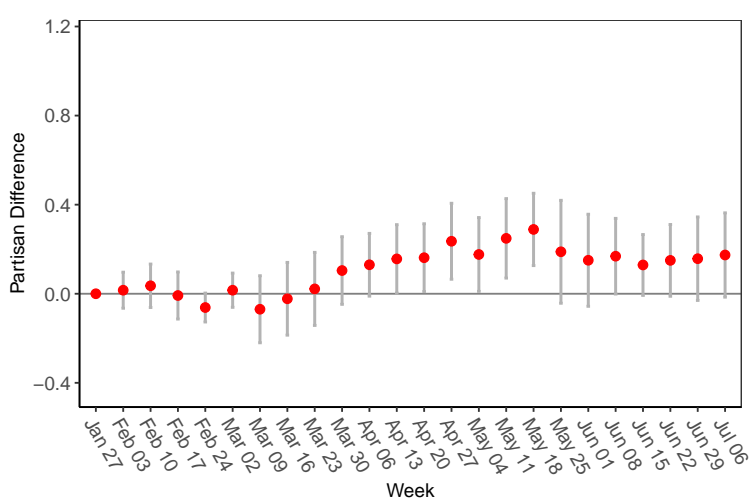

Entertainment

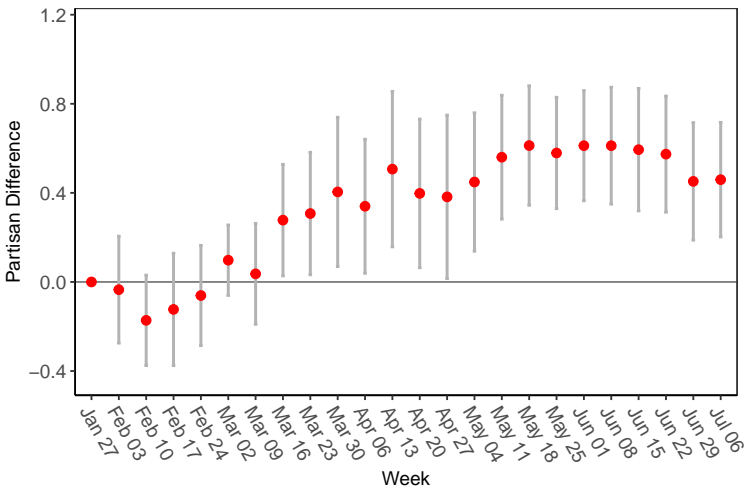

Health Care

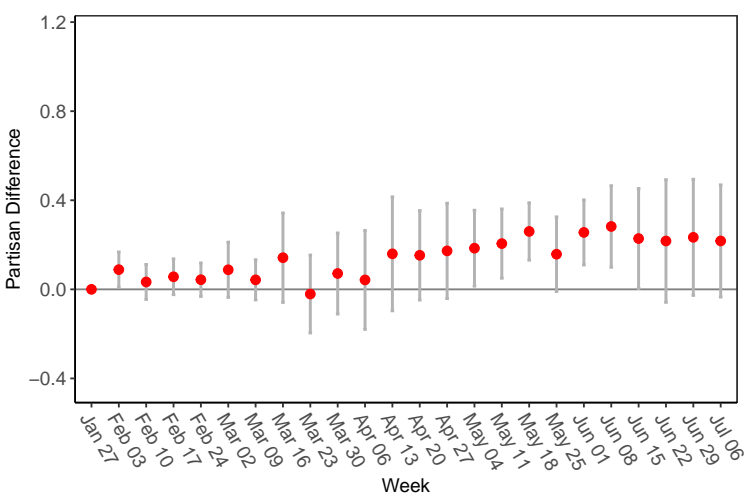

Note: Figure shows the estimated coefficients for county Republican vote share $\rho_{i}$ on the log number of POI visits in the county after restricting POI visits to various 2-digit NAICS codes. The NAICS code groups are: Accommodation and Food (NAICS 72), Entertainment (NAICS 71), Retail Trade (NAICS 44 and 45), Health Care (NAICS 62), and Other Industries (All NAICS codes not previously used). The same controls are used as in Panel C of Figure 4 The grey error bars indicate 95 percent confidence intervals constructed using standard errors clustered at the state-level. 
Appendix Figure A7: Partisan Differences in Social Distancing, Daily

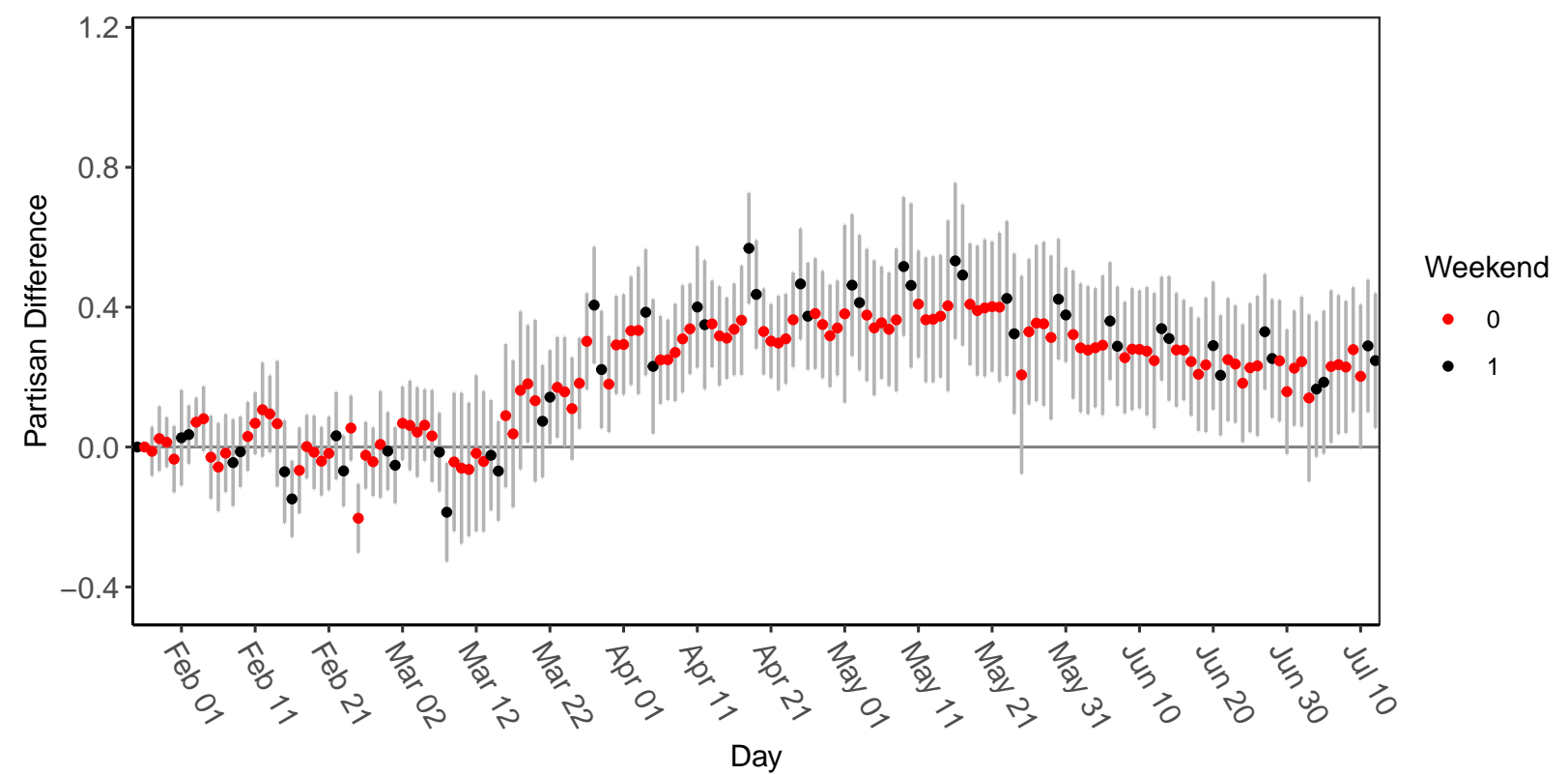

Note: Figure shows the estimated coefficients for county Republican vote share $\rho_{i}$ on the log number of POI visits in the county. The same controls as in Panel C of Figure 4 are used except that state-time fixed effects occur at the day level, and we add separate county fixed effects for weekdays vs. weekends so that weekday and weekend series are normalized separately. The grey error bars indicate 95 percent confidence intervals constructed using standard errors clustered at the state-level. 


\section{Panel A: Alternative Measures by County}

Log Devices Leaving Home

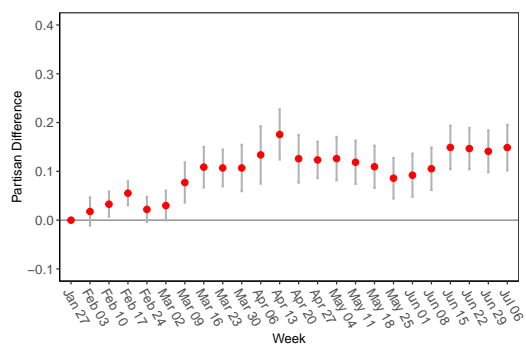

Log Stops in Non-Home CBGs

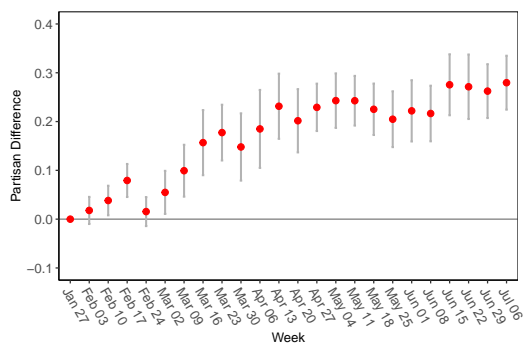

Log Median Time Away from Home

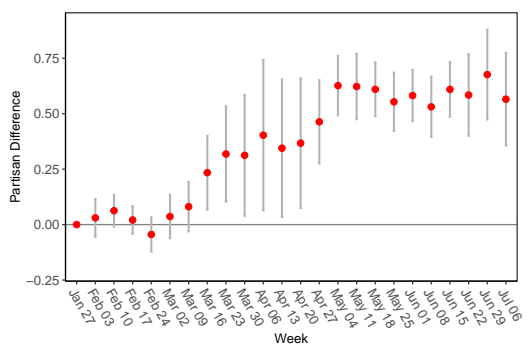

\section{Panel B: Share of Devices Leaving Home by County}

Share of Devices

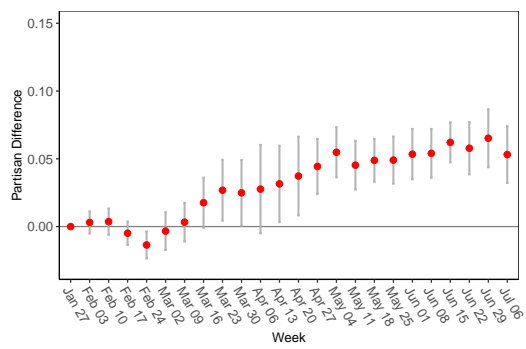

Share of Candidate Devices

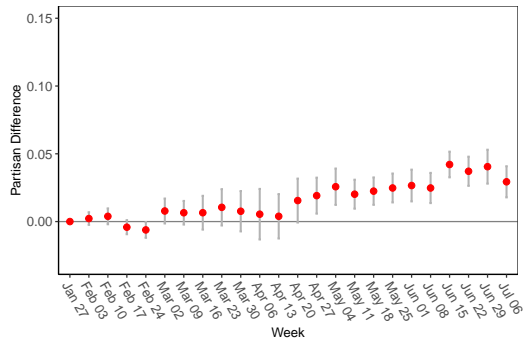

Share Adjusted for Attrition

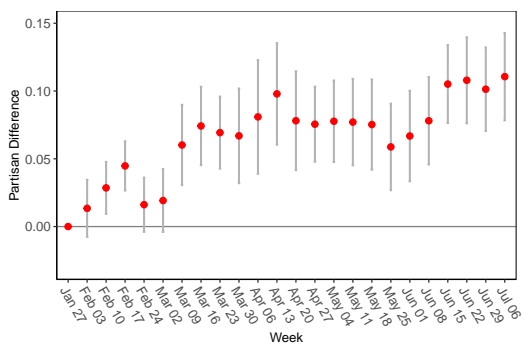

\section{Panel C: Alternative Measures by Precinct}

Log Devices Leaving Home

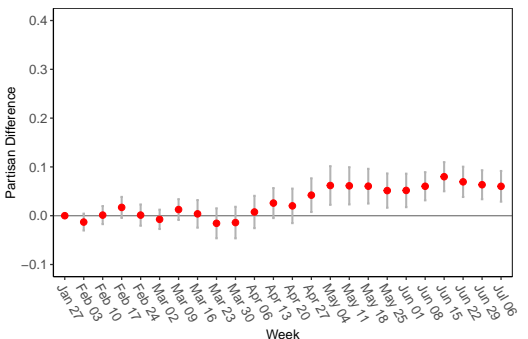

Log Stops in Non-Home CBGs

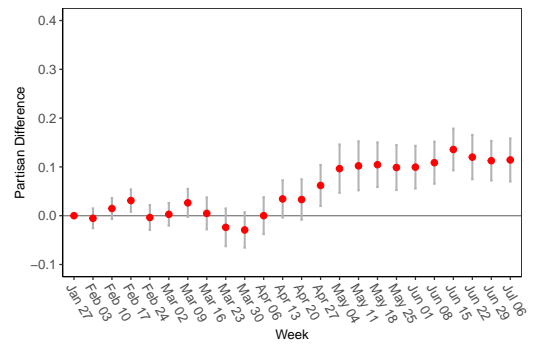

Note: Figure shows the estimated coefficients for Republican vote share $\rho_{i}$ on alternative social distancing measures. The specifications are analogous to our baseline in Panel C of Figure 4 or Panel D of Figure A4 for county- and precinct-level regressions respectively, except that we replace log visits with the following alternative outcomes:

- Panel A: 'Log Devices Leaving Home' is the log of one plus the number of active devices in the panel minus the active devices never observed leaving their geohash-7 home. 'Log Stops in Non-Home CBGs' counts, by home county, the number of devices which stop in a given non-home census block group. We then sum across non-home census block groups and days to form our county-week outcome. 'Log Median Time Away from Home' calculates the median time a device is observed outside its geohash-7 home, by home census block group. We then take a device-weighted average across census block groups and days to form our county-week measure, and then take the $\log$ of this value.

- Panel B: This panel shows the share of devices which are observed outside their geohash-7 home, making different assumptions about attrition observed in the data. 'Share of Devices' is defined to be $1-\frac{\text { home devices }}{\text { current device count }}$, where 'home devices' are active devices never observed leaving their geohash-7 home and 'current device count' is the number of active devices for the current week. 'Share of Candidate Devices' is similarly defined as $1-\frac{\text { home devices }}{\text { candidate device count }}$, where 'candidate device count' is the number of devices regardless of activity.

'Share Adjusted for Attrition' is defined to be $1-\frac{\max \{0, \text { home devices }+ \text { (initital device count-current device count) }\}}{\text { initial device count }}$, where 'initial device count' is the number of active devices for the week of February 1.

- Panel C: This panel produces precinct-level analogues of the first two plots in Panel A. Our specification matches Panel D of Figure A4 (including county-time fixed effects), and we map our original census block group-level social distancing measures to precincts using the method described in Section A.1.2 See footnote 14 for limitations regarding this precinct-level analysis. 


\section{Appendix Figure A9: Partisan Differences in Beliefs and Actions: Unweighted}

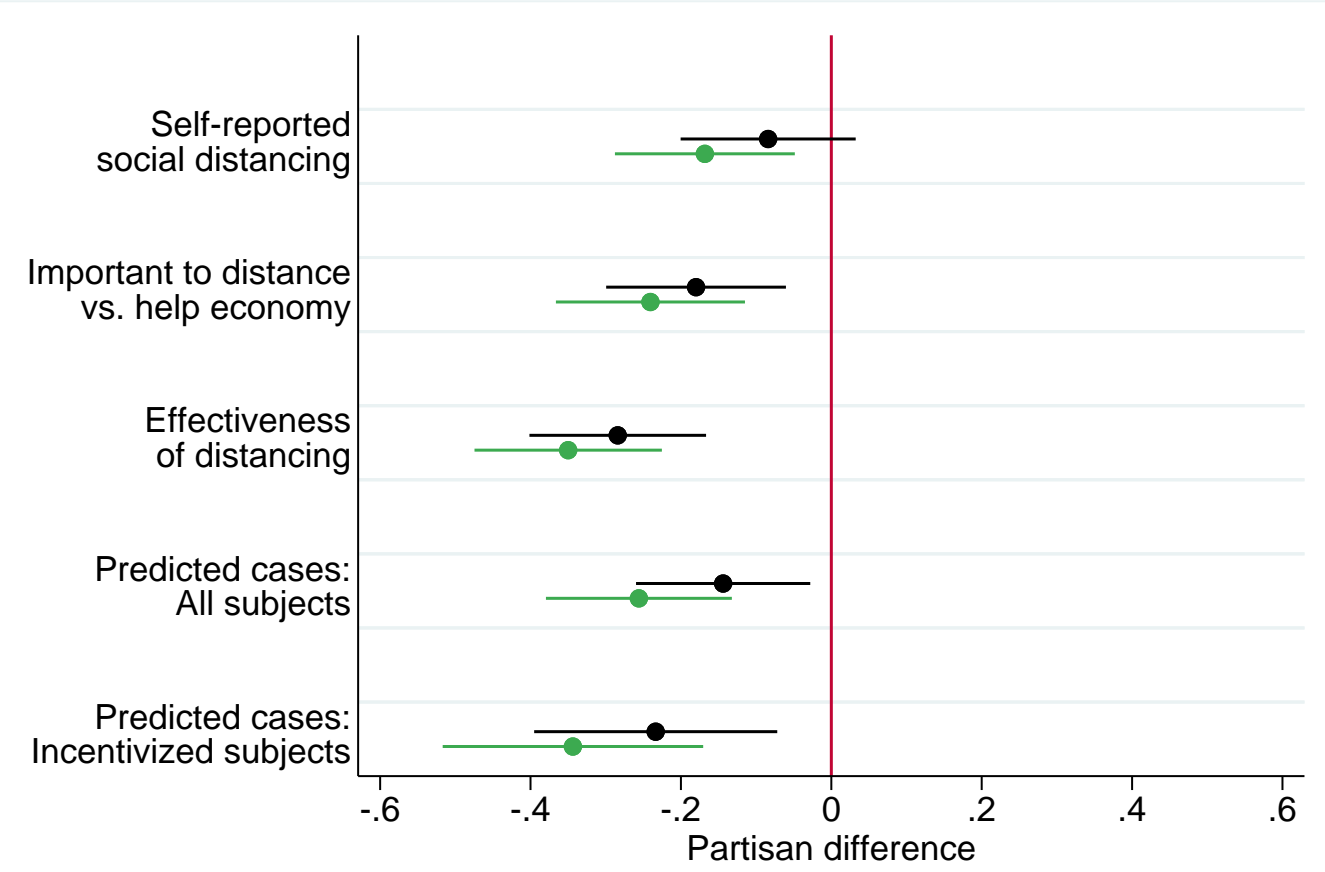

- No controls Controls

Note: Figure shows coefficient plots of regressing normalized measures of beliefs and actions on Republican party lean, without weighting observations. Negative values indicate less concern about COVID-19 or social distancing. Demographic controls are age, race, income, education, number of children, ZIP code logged population density, county-level deaths and cases, and state fixed effects. 2 percent of observations are set to the mean due to an invalid ZIP code. Self-reported social distancing is the percent reduction in contact with others over one month; effectiveness of distancing is the estimated likelihood of catching COVID-19 in one month without social distancing; importance of distancing vs. economy is subjects' perception of whether it is more important to go out and stimulate the economy versus staying in and preventing the spread of COVID-19; predicted cases are predictions about the number of new COVID-19 cases in the U.S. in April; incentivized subjects restrict to the subsample whose answers are incentivized. Error bars represent 95 percent confidence intervals. 


\section{Appendix Figure A10: Effect of Incentives on Beliefs}

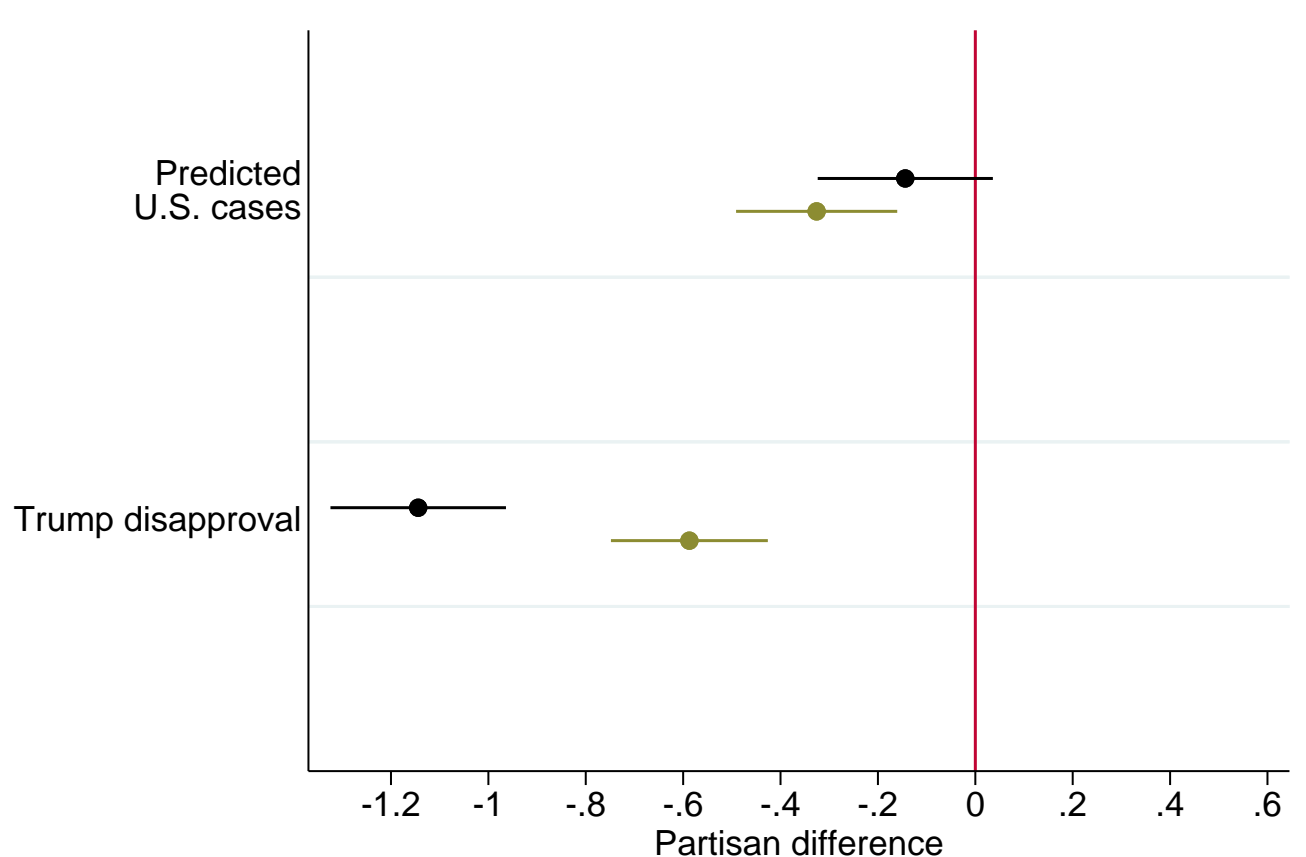

- Unincentivized $\bullet$ Incentivized

Note: Figure shows coefficient plots of regressing beliefs on Republican party lean, with and without incentives for getting close to the correct answer. Trump disapproval is a low-stakes question that is susceptible to partisan cheerleading (Bullock et al. 2015; Prior et al. 2015). These results show that predicting COVID-19 cases does not appear susceptible to the same behavior. Observations are weighted to mimic a representative sample as described in the text. Error bars represent 95 percent confidence intervals. 


\section{Appendix Figure A11: Partisan Differences in Beliefs and Actions: County Fixed Effects}

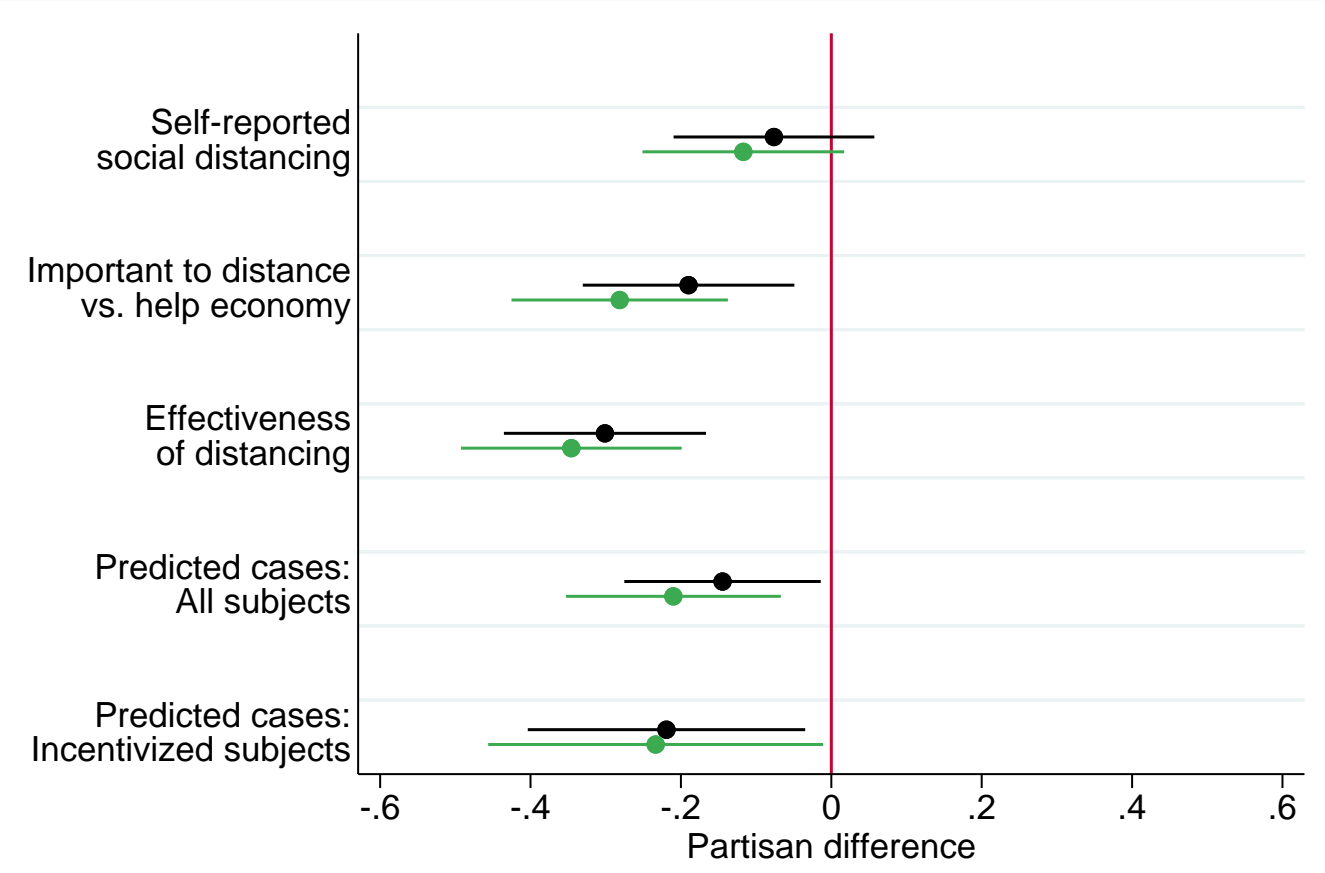

- No controls Controls

Note: Figure shows coefficient plots of regressing normalized measures of beliefs and actions on our seven-point measure of partisan affiliation which ranges between 0 (Strongly Democratic) and 1 (Strongly Republican). Negative estimates indicate less concern about COVID-19 or social distancing. Demographic controls are age, race, income, education, number of children, log population at the ZIP code level, and county fixed effects. 21.5 percent of observations are dropped due to an invalid ZIP code or unique county. The remaining observations are weighted to mimic a representative sample as described in the text. Self-reported social distancing is the percent reduction in contact with others over one month; effectiveness of distancing is the estimated likelihood of catching COVID-19 in one month without social distancing; importance of distancing vs. economy is subjects' perception of whether it is more important to go out and stimulate the economy versus staying in and preventing the spread of COVID-19; predicted cases are predictions about the number of new COVID-19 cases in the US in April; incentivized subjects restrict to the subsample whose answers are incentivized. Error bars represent 95 percent confidence intervals. 


\section{Appendix Figure A12: Differences in Beliefs and Actions by Party and News Consumption}

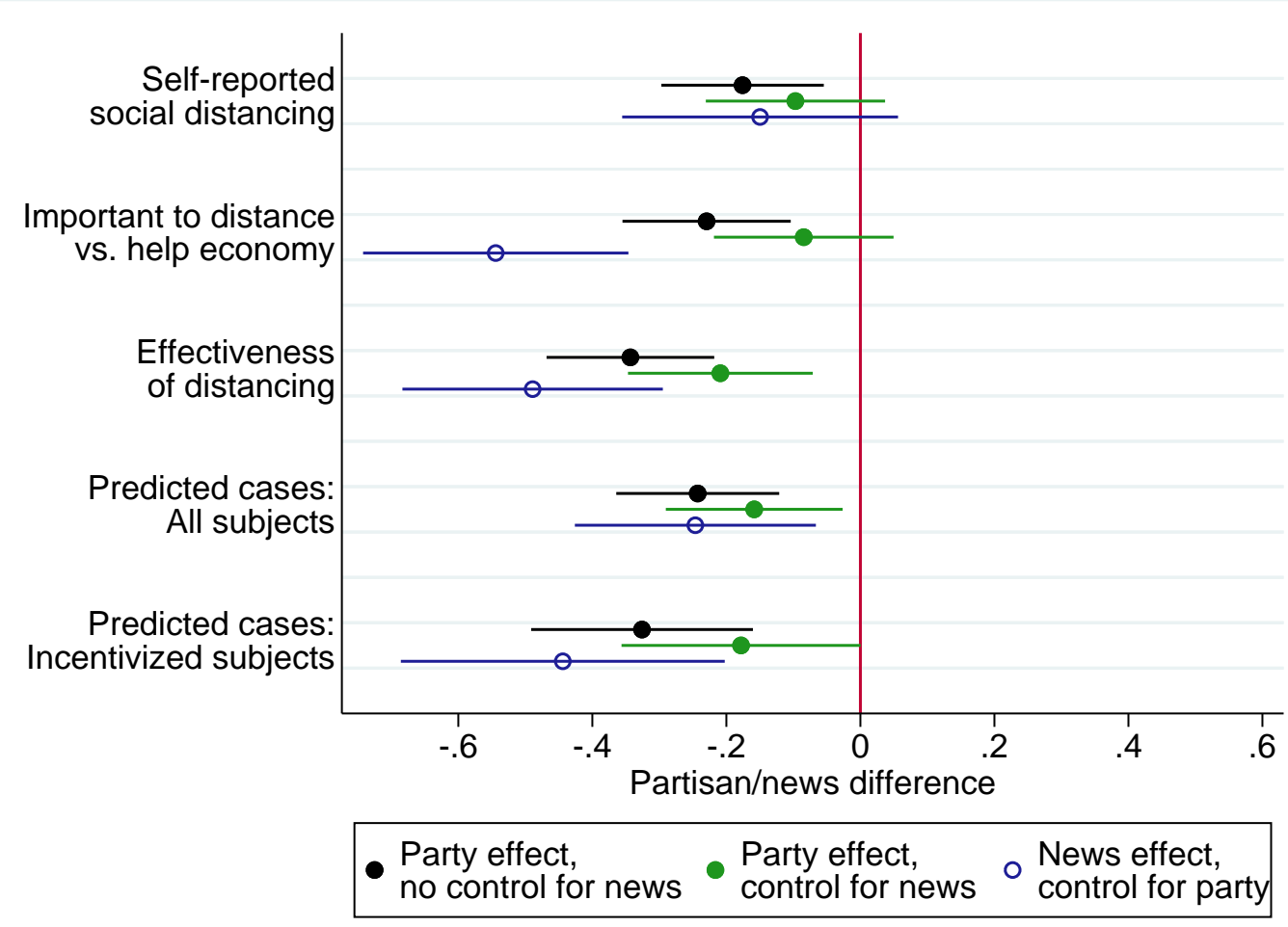

Note: Figure shows coefficient plots of regressing normalized measures of beliefs and actions on coronavirus news consumption and our our sevenpoint measure of partisan affiliation which ranges between 0 (Strongly Democratic) and 1 (Strongly Republican). Negative estimates indicate less concern about COVID-19 or social distancing. News consumption weights respondents' ratings to "How frequently are you getting news and information from each of the following sources about the coronavirus?" as specified in the Survey Details of the Appendix. All coefficients include demographic controls of age, race, income, education, number of children, log population density at the ZIP code level, and state fixed effects. 2 percent of observations are set to the mean due to an invalid ZIP code. For each outcome, the top plot does not control for news, the middle plot linearly controls for news, and the bottom plot shows the coefficient on news in the middle specification. Self-reported social distancing is the percent reduction in contact with others over one month; effectiveness of distancing is the estimated likelihood of catching COVID-19 in one month without social distancing; importance of distancing vs. economy is subjects' perception of whether it is more important to go out and stimulate the economy versus staying in and preventing the spread of COVID-19; predicted cases are predictions about the number of new COVID-19 cases in the U.S. in April; incentivized subjects restrict to the subsample whose answers are incentivized. Observations are weighted to mimic a representative sample as described in the text. Error bars represent 95 percent confidence intervals. 